\title{
Simulations of a Full-Scale Aircraft with Installed Airframe Noise Reduction Technologies
}

\author{
Ryan J. Ferris ${ }^{1}$ \\ Exa Corporation, Burlington, MA, 01803, USA \\ Jason Appelbaum ${ }^{2}$ \\ Exa GmbH, D-70563, Stuttgart, Germany \\ and \\ Mehdi R. Khorrami ${ }^{3}$ \\ Nasa Langley Research Center, Hampton, VA, 23681, USA
}

Computational results are presented for a high-fidelity, full-scale, full-span Gulfstream G-III aircraft model equipped with flap and main landing gear (MLG) noise reduction technologies. The simulations, which were conducted in support of a NASA airframe noise flight test campaign of the same technologies, use the lattice Boltzmann solver PowerFLOW ${ }^{\circledR}$ to capture time-accurate flow data with sound propagation to the far field accomplished using a Ffowcs-Williams and Hawkings (FWH) acoustic analogy approach. The aerodynamic and aeroacoustic behavior of the aircraft were investigated in the approach configuration with combinations of flap and landing gear deployments. The simulated flap concept is an Adaptive Compliant Trailing Edge (ACTE) flap that replaces the Fowler flap system on the G-III aircraft. The simulated MLG noise reduction concept is comprised of porous fairings and a collection of other smaller fairings fitted around the flow-facing components. Using the Fowler flap results as a reference, comparisons are presented on the noise reduction effectiveness of the ACTE flap system. Investigations were made on the effects of using the porous fairings and ACTE flap as noise reduction concepts in tandem. The ACTE flap was found to reduce the total airframe noise level at all flap deflection angles when compared to the Fowler flap equipped model. As anticipated, a reduction in aerodynamic performance was also found when the ACTE flap system was used. The MLG fairings were shown to further reduce the total airframe noise level of the G-III.

\section{Nomenclature}

$\begin{array}{ll}\text { AOA } & =\text { Angle of attack } \\ \mathrm{C}_{\mathrm{L}} & =\text { Lift coefficient } \\ \mathrm{C}_{\mathrm{D}} & =\text { Drag coefficient } \\ \mathrm{C}_{\mathrm{P}} & =\text { Pressure coefficient } \\ \delta_{\mathrm{f}} & =\text { Flap deflection angle } \\ \mathrm{Ma} & =\text { Mach number } \\ \mathrm{Re} & =\text { Reynolds number }\end{array}$

\section{Acronyms}

ACTE $=$ Adaptive Compliant Trailing Edge

CML $=$ Continuous Mold Line

CPUh $=$ CPU Hours

EPNL $=$ Effective Perceived Noise Level

FAA $=$ Federal Aviation Administration

FWH $=$ Ffowcs-Williams and Hawkings

\footnotetext{
${ }^{1}$ Senior Application Engineer, Aerospace.

${ }^{2}$ Aerospace Application Engineer, Aerospace.

${ }^{3}$ Aerospace Engineer, Computational AeroSciences Branch, Associate Fellow AIAA.
} 


$\begin{array}{ll}\text { ICAO } & =\text { International Civil Aviation Organization } \\ \text { LBM } & =\text { Lattice Boltzmann Method } \\ \text { MLG } & =\text { Main Landing Gear } \\ \text { OASPL } & =\text { Overall Sound Pressure Level } \\ \text { PNL } & =\text { Perceived Noise Level } \\ \text { PSD } & =\text { Power Spectral Density } \\ \text { SPL } & =\text { Sound Pressure Level }\end{array}$

\section{Introduction}

A ircraft noise, particularly during the takeoff and landing phases of flight, has an adverse impact on nearby communities and their perception of civil aviation operations. In an effort to reduce this effect, agencies such as the Federal Aviation Administration (FAA) and the International Civil Aviation Organization (ICAO) have established new global noise reduction standards through initiatives to advance research in aircraft noise mitigation [1]. It is generally accepted that system-level aircraft noise has two dominant source types: those stemming from the engine and those from the airframe. Introduction of the modern high-bypass turbofan, in conjunction with the development of engine noise reduction strategies during the last few decades, suggest that any significant decrease in total aircraft noise, specifically during the approach and landing phase, will need to focus on the reduction of airframe sources. The most prominent airframe noise sources are the high-lift (e.g., wing slats and flaps) and the undercarriage systems [2].

The present simulations were performed in support of an extensive flight test campaign conducted under the NASA Integrated Aviation Systems Program Flight Demonstrations and Capabilities (FDC) project, which focused on evaluating the aeroacoustic performance of flap and main landing gear (MLG) noise reduction technologies in a relevant environment. The technologies tested were an Adaptive Compliant Trailing Edge (ACTE) flap that replaces the existing flap system on the test aircraft, and a set of porous fairings and other smaller fairings fitted around the flow-facing components of the MLG.

Leveraging the progress made under the NASA Environmentally Responsible Aviation (ERA) project, the present work and the companion effort in Ref. [3] follow the same computational methodology outlined in Refs. [4], [5], and [6] to perform airframe noise simulations of the full-scale testbed aircraft with and without the noise reduction technologies installed. As an indispensable element of the flight test campaign, the high-fidelity simulations were used in two ways. First, they were essential during tailoring and retrofitting of the gear fairings into the main landing gear of the NASA Armstrong Flight Research Center (AFRC) SubsoniC Research Aircraft Testbed (SCRAT) [7], a Gulfstream G-III business jet. The fairings, which were originally developed for testing on the larger Gulfstream G$\mathrm{V}$ aircraft, had to undergo significant design alterations in order to fit into the smaller and more compact G-III main landing gear [7] [8]. During this initial stage, after each major design change was completed, the aeroacoustic performance of the redesigned fairings was evaluated via simulations of the full-scale landing gear installed on a semispan model of the G-III aircraft to ensure that the noise reduction effectiveness of the fairings was not seriously compromised. Important indicators used to judge fairing effectiveness were the level of reduction in gear surface pressure fluctuations that had been achieved and whether the regions showing elevated unsteady pressures had been eliminated or diminished substantially. During each iteration cycle, key observations and trends from the simulations were communicated to the AFRC design engineers who, in turn, incorporated appropriate (albeit modest) changes to the fairings that helped optimize their acoustic performance while maintaining structural, gear operation, brake cooling, and a host of other design requirements.

The second major way in which the simulations contributed to the success of the flight test campaign was by providing the capability to conduct system-level, full-scale evaluations of the flap and gear noise reduction hardware prior to the test. The insights gained from the simulated results were instrumental for the execution of a more productive test campaign, since the focus could be shifted to those aircraft conditions and configurations that yielded stronger trends for evaluating the performance of the technologies. Moreover, this integrated and synergistic execution of the flight test and simulations afforded us the opportunity to perform direct comparisons between measured and simulated aeroacoustic results in order to validate the predictions and determine the strengths and shortcomings of the computational methodology used in this study.

\section{Aircraft Configuration and Flight Conditions}

The model developed for the present simulations is a full-scale, full-span, high-fidelity representation of the SCRAT aircraft with ACTE flaps installed [7]. The model comprises a fuselage, wing, ACTE flaps, flow-through nacelles including an engine hush kit, pylon, and main landing gear. The aircraft fuselage and the wings with the 
ACTE flaps are modified versions of those used in Refs. [9] and [10] to calculate steady aerodynamic loads for the aircraft without the landing gear. Clearly, the fidelity of any airframe noise prediction is highly dependent on how well the local geometric details are captured and represented in the simulations. Thus, extra time was devoted to recreate many of the streamwise and spanwise slits, cavities and openings, dips and bumps, that are present on the aircraft surface in regions where the ACTE flaps transition and mate with the main wing. The surface definition for the hush kit, which is a recent addition to the G-III engines, was developed from information and engineering drawings provided by the manufacturer and from physical inspection of the aircraft.

A highly defined geometry of the main landing gear, including its cavity, was painstakingly developed from carefully conducted laser scans of the component while the aircraft was placed on jacks to have the gear in the fully extended landing configuration. Significant effort was spent ensuring that the finest details of the main gear exterior surfaces (i.e., outer mold lines) were captured accurately. However, a major deficiency of this type of geometry reconstruction is the lack of proper definition for some of the internal cavities that may be present on some of the gear sub-elements, which could potentially contribute to the tonal component of the gear noise signature. Fortunately, for the G-III main landing gear, only the opening in the front post, which runs the entire length of the component, could not be mapped out with the laser scanning process. The internal opening in this post was reconstructed via direct measurements of a few critical locations and features of this gear component. As a result, the frequency of the predicted tone generated by the post cavity was noticeably different than the frequency measured during the flight test - a direct result of the mismatch between modeled and "as flown" volume of the opening in the front post of the main gear [8]. A description of the modeled baseline G-III aircraft with its original Fowler Flap system is provided in the companion paper [3].

\section{A. Aircraft Configuration}

As described in Refs. [9], [10], and [11], the ACTE flap replaces both of the G-III's conventional 19-foot-long aluminum Fowler flaps with advanced, shape-changing flaps that form continuous bendable surfaces. A side-by-side comparison of the ACTE and Fowler flap configurations can be seen in Fig. 1. At the juncture between the flap and the main wing body, the ACTE system has an elevated cover on both pressure and suction sides of the wing. After this cover, there is a smooth contour of changing radius until its termination at the trailing edge. At the inboard and outboard ends of the flap, transition sections allow continuous mating of the flap to the adjacent main wing segments, thus eliminating the side-edges found on a typical Fowler flap configuration. Slightly beyond the flap transition regions, there is a small gap extending the streamwise length of the flap that will be referred to as the flap slit. A more detailed view of notable ACTE and Fowler flap system features can be seen in Fig. 2.

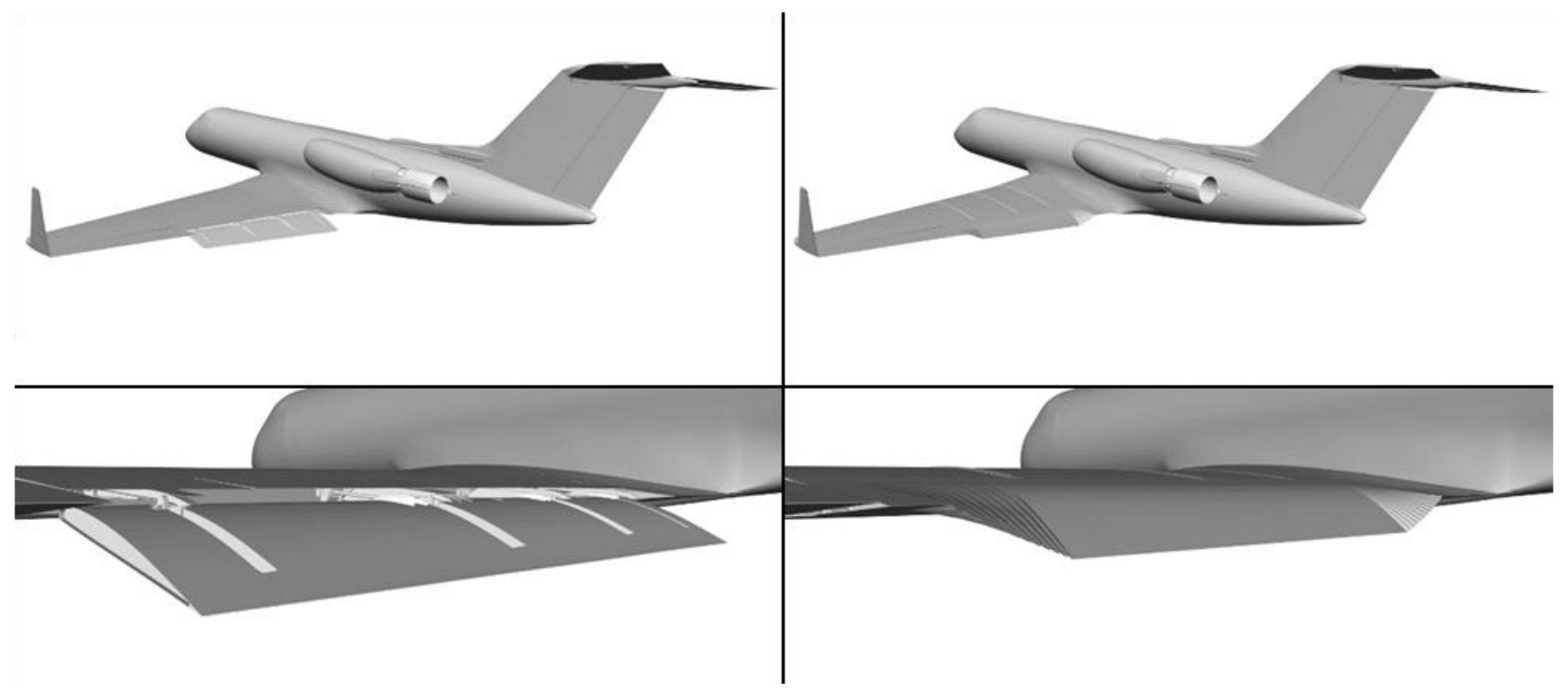

Fig. 1 Side-by-side comparison of Fowler $\left(\delta_{\mathrm{f}}=20^{\circ}\right.$, left $)$ and ACTE $\left(\delta_{\mathrm{f}}=25^{\circ}\right.$, right $)$ flap configurations, without MLG. 


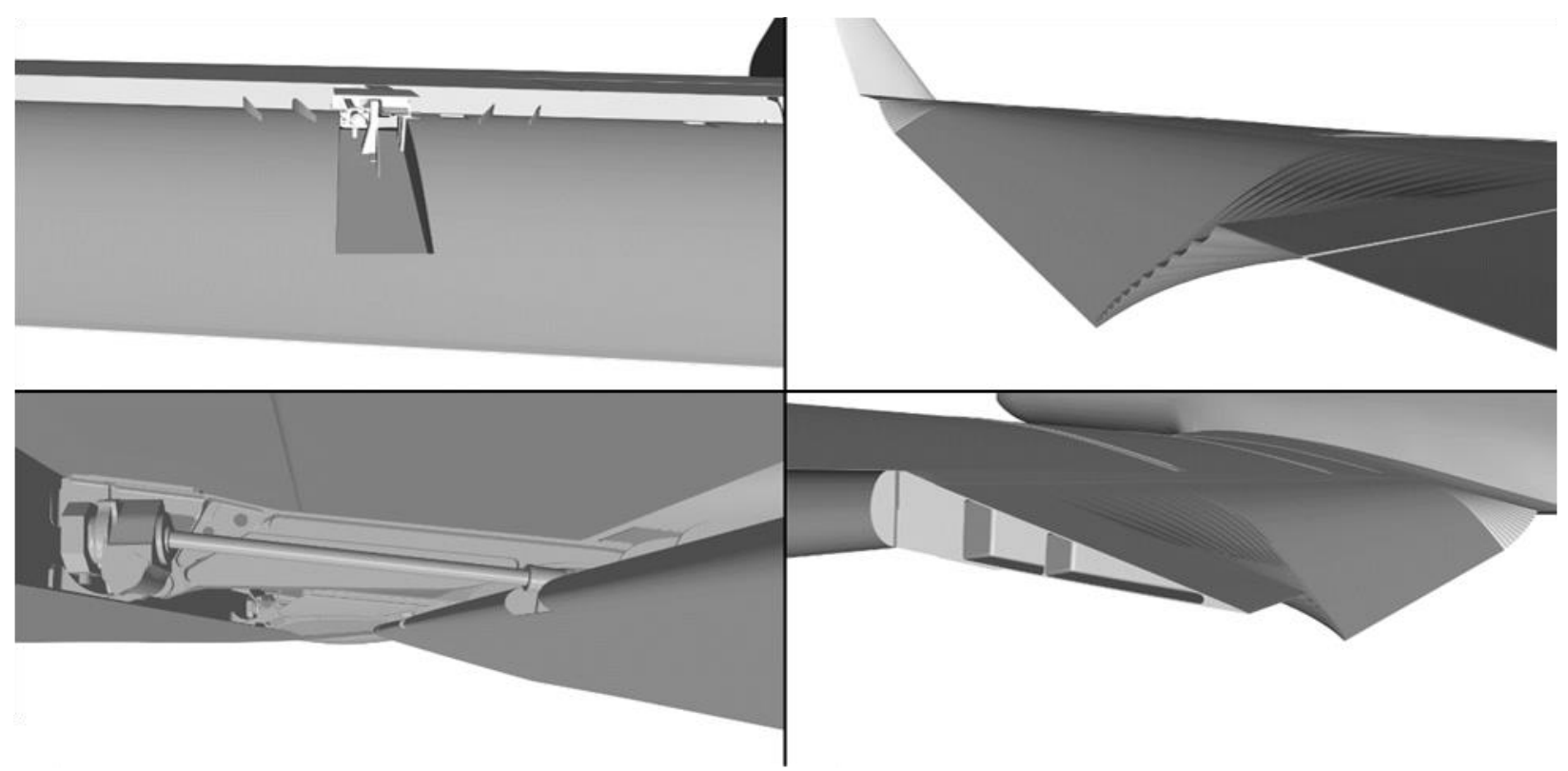

Fig. 2 Notable features of the Fowler $\left(\delta_{\mathrm{f}}=20^{\circ}\right.$, left $)$ and ACTE $\left(\delta_{\mathrm{f}}=25^{\circ}\right.$, right $)$ flap systems. Clockwise from top left: Exterior view of Fowler flap track region; close-up view of the inboard ACTE mold line; view from underneath the wing toward the wing tip, into the Fowler flap cove; and view of ACTE flap side-edge cavities with aileron removed.

Comparisons of the G-III MLG with and without the fairings can be found in Fig. 3. The fairings comprise the porous knee fairing (PKF) covering the front post plus an assortment of smaller fairings that are collectively referred to as upper fairings (UF). The porous knee fairing is composed of lower and upper sections. The lower segment extends from the juncture of the torque tubes to the bottom of the lower shock tube and is form-fitted to allow clearance between the fairing and the wheels. To maintain the desired open-area-ratio there are 3,597 $2 \mathrm{~mm}$ (0.080 in) holes extending through the fairing body on the forward face of the lower segment. The upper segment of the porous fairing is wider, and like the lower fairing, is restricted from extending too far aft by the need for proper wheel clearance. On the upper segment of the PKF, there are 7,735 $2 \mathrm{~mm}$ holes extending through the body. In total, there are 11,332 2 $\mathrm{mm}$ holes on the two PKF segments that were simulated as part of this investigation. The UF is comprised of three smaller fairings. The inboard close-out fairing, which resides above the PKF and is form-fitted around the underlying components, includes a retract strut cap fairing where the side strut attaches to the upper torque tube. The inboard close-out fairing is not porous. The UF also includes a teardrop shaped door strut fairing.

The G-III MLG, along with the landing gear bay, are shown in Fig. 4. The landing gear bay is embedded within the center of the main wing body, just under the fuselage and between the main landing gear structures. The computational model of the MLG bay is a hollow multichamber cavity, symmetric across the aircraft centerline, with the main identifying feature of each chamber being the vertical penetration into the aircraft. Spanning the centerline of the cavities is a small solid body used to represent the blockage that is typically present in this section of the landing gear bay. Absent from the modeled landing gear bay is the multitude of hydraulic hoses, tanks and other minutia that are typically found in this region of a physical aircraft. The exclusion of these components was a decision reached after weighing the difficulty in laser scanning each of these small parts with the relative lack of effect they would have on the overall acoustic results. Early simulation results showed that a powerful resonant cavity response was excited by the unsteady shear layer grazing the cavity opening. To increase the effectiveness of assessing flap and MLG lownoise concepts, the acoustic dampening effect of the missing components was modeled, as described in Ref. [3], by way of increasing the relative fluid viscosity of a thin layer adjacent to the MLG bay ceiling.

\section{B. Flight Conditions}

The executed simulations can be grouped into two main categories. The first group represents those computations that were performed in support of the preliminary and critical design reviews (PDR and CDR, respectively) of the main gear noise reduction hardware, both of which took place prior to commencing the flight test campaign. Not knowing a priori the exact angle-of-attack (AOA) of the testbed aircraft during its passes over the microphone array and given the weak dependency of the airframe noise sources on AOA in the linear lift regime [12], this group of 
simulations was conducted at a fixed $\mathrm{AOA}=6^{\circ}$, which is within the range of most landing operations. The Reynolds number $(\mathrm{Re})$ for all computations was set at $10.5 \times 10^{6}$, representing a value that is close to $60 \%$ of the flight Re based on the mean aerodynamic chord of $13.78 \mathrm{ft}(4.2 \mathrm{~m})$ and an aircraft speed of 150 knots. This chosen Re is sufficiently high to produce farfield noise levels that are nearly equivalent, at a lower computational cost, to those obtained at full flight Re [6]. A detailed discussion on the simulation flow parameters and conditions can be found in the companion paper [3].

The second group of simulations comprises those that were conducted after the first flight test campaign was executed during August-October 2016. For these simulations, the AOAs were chosen to closely match aircraft parameters recorded during the microphone array flyovers.

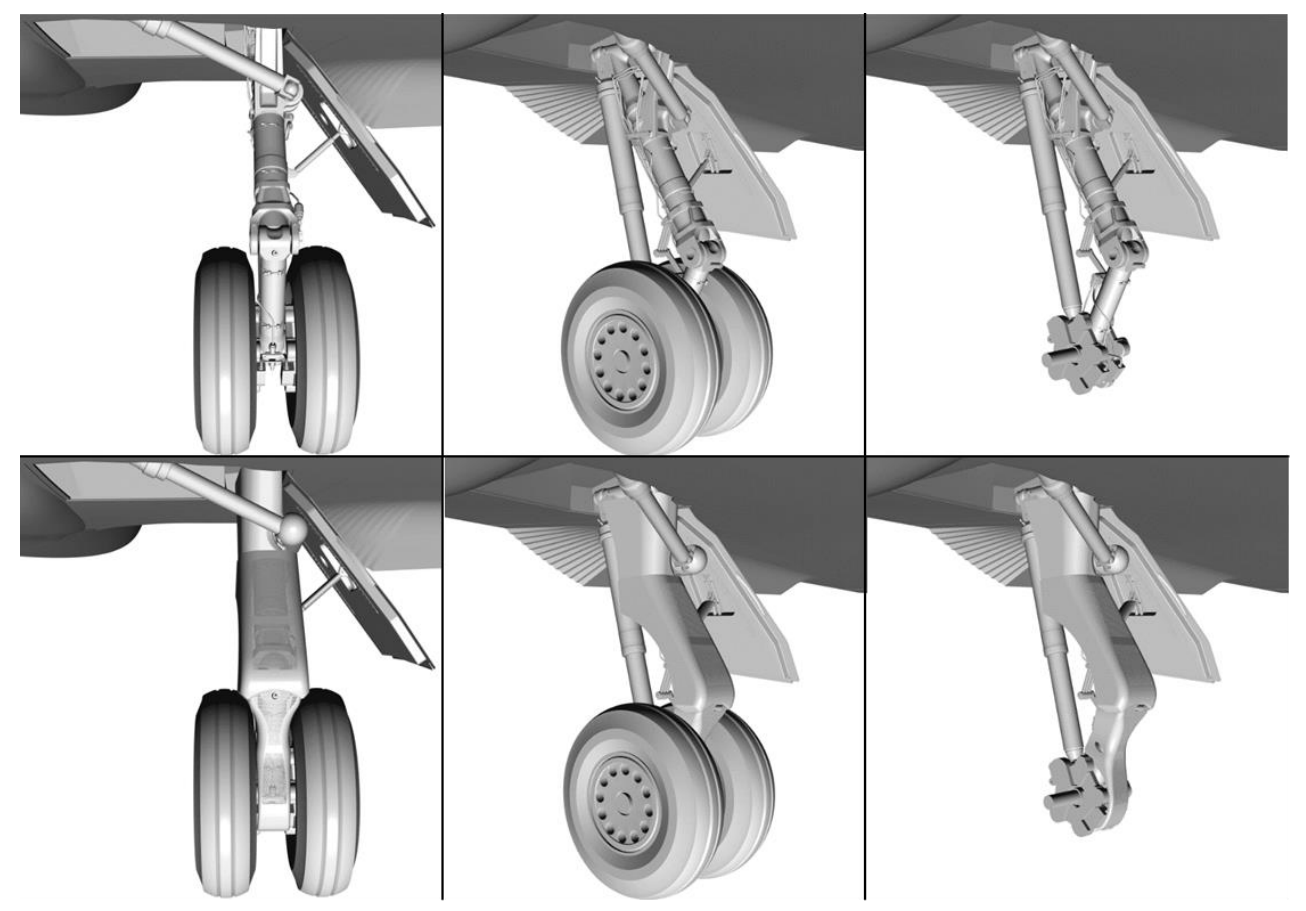

Fig. 3 Gulfstream G-III with and without MLG fairings. Upper row shows the port-side MLG without fairing. Lower row shows the port-side MLG with fairing. Far right images have the wheel assembly removed to show detail in this region.
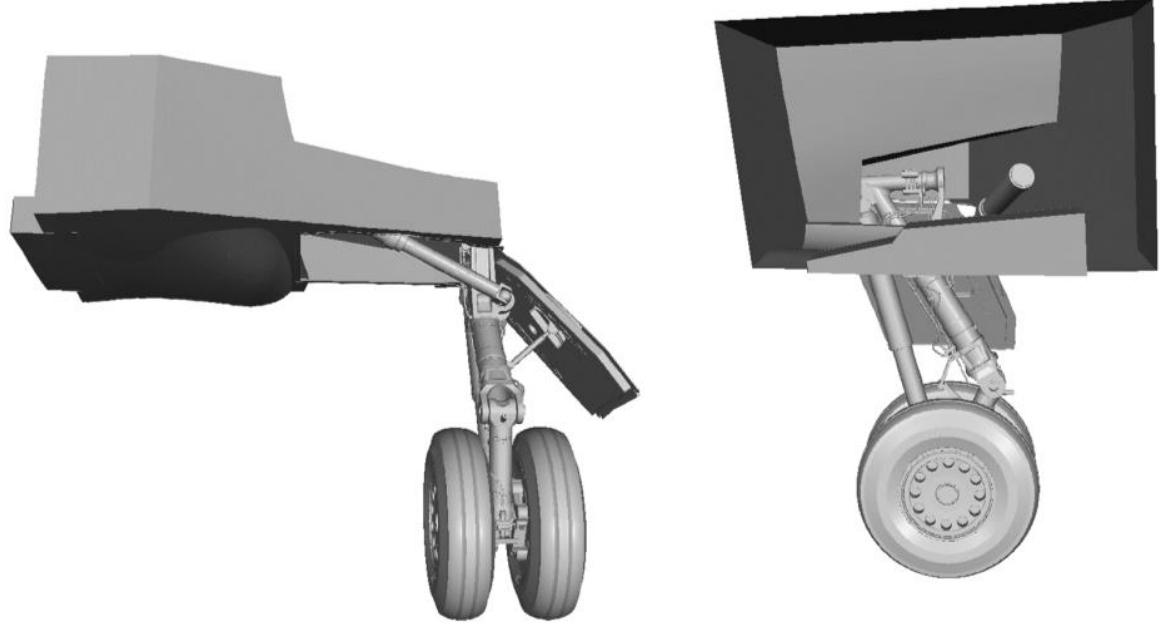

Fig. 4 Gulfstream G-III port-side MLG and bay cavity. 


\section{Computational Approach}

The numerical simulations were performed using Exa Corporation's PowerFLOW ${ }^{\circledR}$ solver, which is based on the three dimensional 19 state (D3Q19) lattice Boltzmann method (LBM) [13] [14] [15] [16]. LBM has been extensively validated for a wide variety of applications ranging from academic direct numerical simulation (DNS) cases to industrial flow problems in the fields of aerodynamics [17] and aeroacoustics [4] [18] [19]. At a macroscopic level, LBM uses a simpler and more general physics formulation than methods based on the Navier-Stokes equations [13]. The LBM equations recover the macroscopic hydrodynamics of the Navier-Stokes equations through the ChapmanEnskog expansion [20] [21].

The local formulation of the LBM equations allows a highly efficient implementation for distributed computations on thousands of processors. The low dissipation and dispersion properties of the numerical scheme typically produce aerodynamic and aeroacoustic results that are comparable to those obtained with classical CFD solvers that use higherorder large eddy simulation (LES).

\section{A. Turbulence Modeling}

The lattice Boltzmann flow simulation is equivalent to a DNS of the flow. For high Re flows, such as those addressed in this work, the lattice Boltzmann Very Large Eddy Simulation (LB-VLES) approach is used to reduce computational resource requirements [13] [22]. This means that turbulence is modelled in areas of attached flow such as boundary layers but resolved in wakes or regions of detached flows.

\section{B. Wall Treatment}

The standard lattice Boltzmann bounce-back boundary condition for no-slip or the specular reflection for free-slip condition are generalized through a volumetric formulation [13] [14] near the wall for arbitrarily oriented surface elements (surfels) within the Cartesian volume elements (voxels). This formulation of the boundary condition on a curved surface cutting the Cartesian grid is automatically mass, momentum, and energy conservative while maintaining the general spatial second-order accuracy of the underlying LBM numerical scheme. To reduce the resolution requirements near the wall for high Re flows, a hybrid wall function is used to model the region of the boundary layer closest to the solid surfaces [17] [23].

\section{Simulation Setup}

The present simulations were performed at full scale and with free-air boundary conditions. A freestream Mach number (Ma) of 0.23 and Re of $10.5 \times 10^{6}$ based on mean aerodynamic chord were used for the computations. The simulation domain was initialized with free flow conditions except in the immediate nearfield region around the aircraft, which was initialized with zero flow velocity and freestream pressure. For the first several cases run, a symmetry plane normal to the lateral direction was used to reduce computational cost. The effect of using the symmetry plane is discussed in Ref. [3].

The initial $\sim 0.60 \mathrm{~s}$ of simulated physical time represents the transient to settled flow conditions and is not used for statistical sampling. After this initial time segment, various data are recorded from the simulation, most importantly the high-frequency sampled pressure field on the surface of the aircraft and the density, velocity and pressure field on a permeable surface outside of the acoustic near field. These datasets are written out for $\sim 1.50 \mathrm{~s}$ of simulated physical time. These two main measurements are eventually used in conjunction with Exa's farfield noise solver within PowerACOUSTICS ${ }^{\circledR}$ to obtain pressure signals at defined locations.

\section{Data Acquisition}

The main acoustic data for comparison in this paper were obtained from the solid surface pressure field, which is comprised of a time-accurate pressure history on the aircraft surface. A detailed description of the measurement surfaces and data acquisition procedure can be found in Ref. [3]. For the purposes of this paper, all comparative acoustic analyses were done using this solid measurement surface, as explained in section IV-B.

\section{F. Farfield Noise Solver}

An acoustic analogy approach based on the Ffowcs-Williams and Hawkings (FWH) formulation [24] was used to propagate the computed nearfield fluctuations to the far field via the efficient and well-validated formulation developed by Farassat [25], also known as formulation 1A. The formulation is extended to account for uniform mean flow convection effects to simulate the noise generated and measured in an ideal infinite wind tunnel [26].

\section{G. EPNL}

The Effective Perceived Noise Level (EPNL) was calculated using a proprietary post-processing tool developed by Exa Corporation. The primary reference for the definition and calculation of EPNL is a publication of the ICAO [27]. The input to the tool is a semicircular arc of FWH signals. Once a trajectory is determined, it is discretized into flight segments of a given duration, in this case $500 \mathrm{~ms}$. For every flight segment, the emission time and position of 
the aircraft is determined, and a ray between the observer and aircraft is traced. The ray's intersection with the microphone array on the ground is determined, and a noise spectrum is formulated by interpolating the appropriate microphone narrow-band spectra. The spectra at the observation point are corrected for distance, atmospheric absorption, Doppler shifting and ground reflection for each flight segment. One-third octave sound pressure levels (SPL) are then computed and Perceived Noise Level (PNL) is calculated using the procedure described by the ICAO. Finally, tonal weighting and band sharing adjustments are made, and EPNL is computed.

\section{Numeric Results}

\section{A. Grid Resolution Study}

A detailed mesh resolution analysis was performed as part of this study, the process of which is identical to that described in the companion paper of Ref. [3]. The behavior of the solution as the resolution increased was comparable between the simulations with porous fairings to those without the porous fairings. Therefore, this section will focus on results for the ACTE flap configuration with MLG deployed but without the porous MLG fairings.

From an aerodynamic standpoint, we will review the streamwise surface pressure contours in Fig. 5, which are positioned at points corresponding to the location of the pressure strips used during the flight test. These will be referred to as inboard flap, midflap, and outboard flap (corresponding to spanwise locations of $136 \mathrm{in}, 201 \mathrm{in}$, and 269 in, respectively) for the purposes of this section. The rapid changes in $\mathrm{C}_{\mathrm{P}}$ at $10 \%$ and approximately $80 \%$ of the chord in each plot are attributable to the sudden change in surface contour moving from the surface of the wing to that of the applied pressure strip and then back to the surface of the wing. As resolution was increased from coarse through fine, a number of trends were identifiable. First, the minimum $C_{P}$ on the suction side of the flap increased at the inboard location and decreased at the mid and outboard flap locations. The point of flow separation on the surface of the flap translated toward the leading edge in all but the inboard flap section, indicating that a larger portion of the flap surface was underneath separated flow. Lastly, much of the wing surface was relatively unchanged between resolution levels. Especially of note is the leading-edge suction peak, a region of high gradients and therefore sensitive to resolution changes.
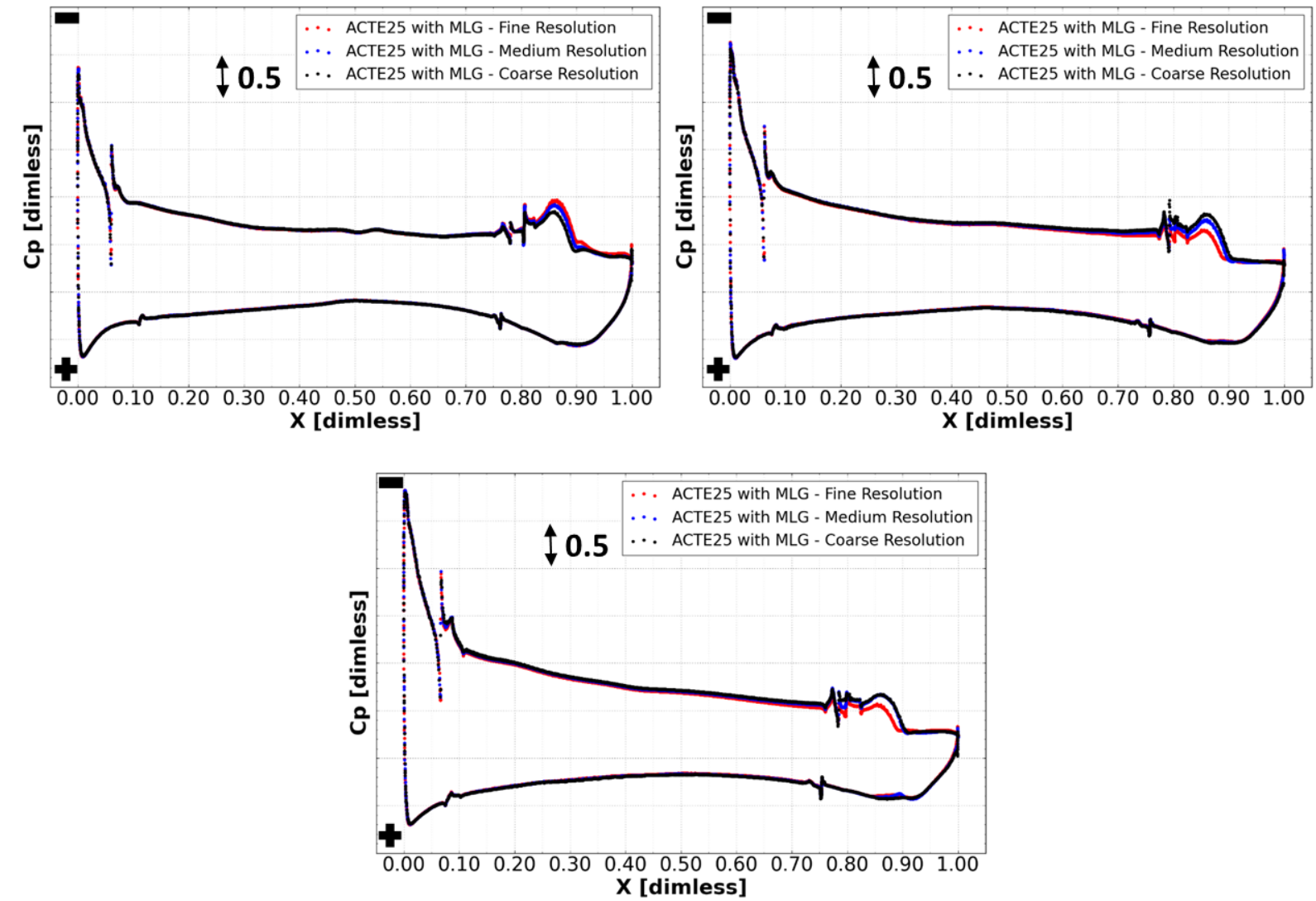

Fig. $5 \mathrm{C}_{\mathrm{P}}$ profiles comparing the $\mathrm{ACTE}\left(\delta_{\mathrm{f}}=25^{\circ}\right)$ flap configurations at the given resolution levels. Top Left: inboard pressure strip. Top Right: midflap pressure strip. Bottom Image: outboard pressure strip. 
A contour plot of static pressure on the surface of the aircraft is shown in Fig. 6. In this figure, the influence of the pressure strips on the upper surface of the flap is visible, causing discontinuities in the streamlines just aft of their extents. Also observed from Fig. 6 are 1) the diffusion of the three-dimensional separation regions (whose imprints on the flap surface are manifested via large spiral structures) as the resolution goes from coarse to fine, and 2) a global reduction in minimum $C_{P}$ on the surface of the flap, except near the inboard section where the absolute magnitude of this value has increased. Large separation zones such as those encountered on the current ACTE flap evolve based on long periods (very low frequencies), in particular when the spatial resolution is increased, producing finer flow structures within the separation zone. As a result, the flap suction peaks at the three extracted spanwise locations may increase or decrease if the simulation record is extended beyond the current $1.5 \mathrm{~s}$ to generate a longer record before time-averaged quantities are extracted.

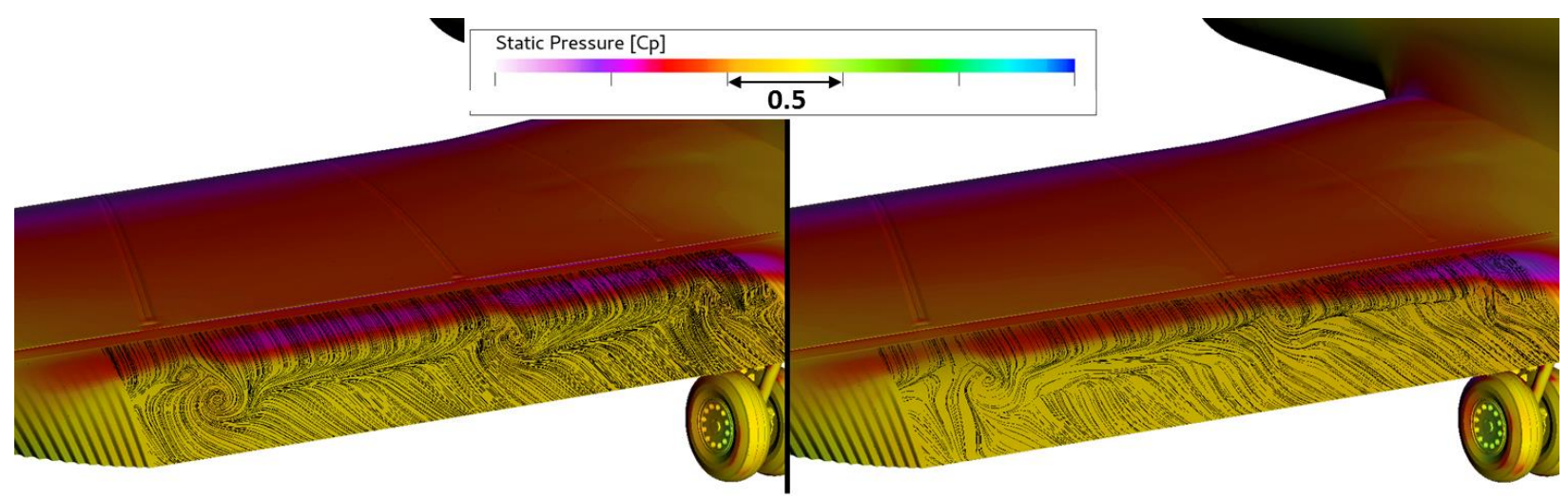

Fig. 6 Time-averaged pressure distributions showing coarse (left image) and fine (right image) resolution results with streamlines on the flap surface.

The behavior of the acoustic results with changing resolution is given in Fig. 7. From these datasets, a clear distinction is visible among resolution levels. The spectra on the right image show good fine-to-medium resolution convergence up to frequencies around $1500 \mathrm{~Hz}$, at which point a deviation begins to appear and slowly grow. With the increase in resolution, a decrease in voxel size is realized thereby allowing more voxels per wavelength at a given frequency. This spatial resolution is important in the higher frequencies as wavelength becomes smaller, requiring smaller voxels to adequately resolve the propagating wave. The increase in PNL $\operatorname{MAX}$ (left image) appears to be relatively constant with each subsequently finer resolution level simulated. As a metric, PNL is greatly influenced by the high frequency content present in a signal [29]. Thus, higher PNL levels are proportional to the increased high frequency content resolved in the finer resolution simulations.
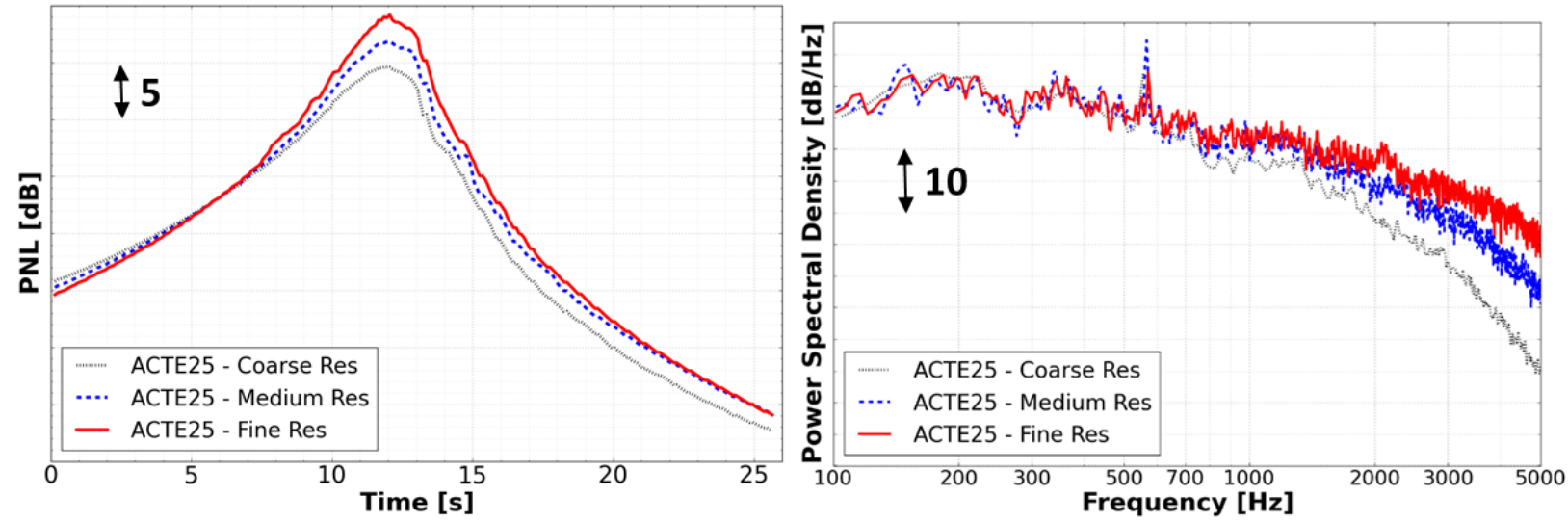

Fig. 7 Acoustic comparisons at the given resolution levels. Left Image: PNL vs. Time. Right Image: PSD plotted at the $90^{\circ}$ directivity.

Some insights regarding computational resource trends between configurations and resolution levels can be gleaned from Tables 1 and 2. Both tables present metrics for the ACTE 25 configuration at each resolution level 
simulated, with Table 1 providing details for the cases without porous MLG fairings and Table 2 for the cases with porous MLG fairings. The CPU hours (CPUh) listed are an accurate approximation, based on performance realized during the study, of what one could expect running these same simulations without any cluster related issues. To properly capture the flow through the small holes within the porous MLG fairings, an additional level of resolution was required. This is reflected in the relative differences between the minimum edge as well as the increase in timesteps between configurations. A difference in number of timesteps also exists between the coarse and medium simulations compared to the work presented in Ref. [3]. This is attributable to a reduction in the initial transient time needed for the ACTE configuration compared to that of the Fowler flap configuration. Note also that the doubling in timesteps between the two fine cases listed did not lead to an equivalent increase in CPUh. PowerFLOW ${ }^{\circledR}$ solves for the finest level of resolution every time step, the next finest level of resolution every other timestep, and continues that pattern throughout the resolution levels in a given simulation. In the cases with porous MLG fairings, the finest level of resolution is reserved for the small holes, thereby occupying relatively less fluid volume and requiring relatively fewer number of voxels to be solved for each timestep.

Table 1 Simulation metrics for the ACTE configuration resolution study: ACTE25 with MLG deployed.

\begin{tabular}{lcclccc} 
Resolution & Voxels $\left[10^{6}\right]$ & Surfels $\left[10^{6}\right]$ & Min Edge $\left[10^{-4} \mathrm{~m}\right]$ & Timesteps & \# Processors & $\sim$ CPUh $\left[10^{6}\right]$ \\
\hline Coarse & 1084 & 57 & 7.2 & 2293760 & 3000 & 0.21 \\
Medium & 3256 & 103 & 4.8 & 2719744 & 4000 & 0.85 \\
Fine & 15104 & 200 & 3.2 & 4063232 & 10000 & 4.93 \\
\hline
\end{tabular}

Table 2 Simulation metrics for the ACTE configuration resolution study: ACTE25 with MLG deployed and porous fairings installed.

\begin{tabular}{lccllcl} 
Resolution & Voxels $\left[10^{6}\right]$ & Surfels $\left[10^{6}\right]$ & Min Edge $\left[10^{-4} \mathrm{~m}\right]$ & Timesteps & \# Processors & $\sim$ CPUh $\left[10^{6}\right]$ \\
\hline Coarse & 1446 & 97 & 3.6 & 3604480 & 3000 & 0.84 \\
Medium & 4792 & 168 & 2.4 & 5406720 & 5000 & 1.63 \\
Fine & 15620 & 312 & 1.6 & 8126464 & 10000 & 6.40 \\
\hline
\end{tabular}

\section{B. Solid vs. Permeable Measurement Results}

Two types of measurement surfaces, as outlined in Section III(D), were recorded during simulation for acoustic analysis purposes. As indicated in Ref. [3], a judiciously placed permeable measurement surface and not a solid measurement surface corresponding to the aircraft skin produced the most accurate results when compared to direct probe measurements taken in the same simulation. Throughout the course of this study, alterations were made to the shape of the permeable surface in an attempt to arrive at what would be the best tradeoff between suitable frequency resolution and overall cost limitation. This meant that somewhat different permeable surfaces were used for some simulations, precluding the execution of direct comparisons. The solid measurement surface remained consistent throughout the simulations presented in this paper and thus permitted more representative conclusions to be drawn. To demonstrate the differences between the solid and permeable measurement surfaces resulting from the ACTE configuration, we will first introduce the optimum permeable surface identified during this study as well as examine some acoustic comparisons. The permeable surface shown in Fig. 8 is this optimum shape, which resulted from combining the shape studied in Ref. [3] with semiplanar endcaps to allow for hydrodynamic filtering. 

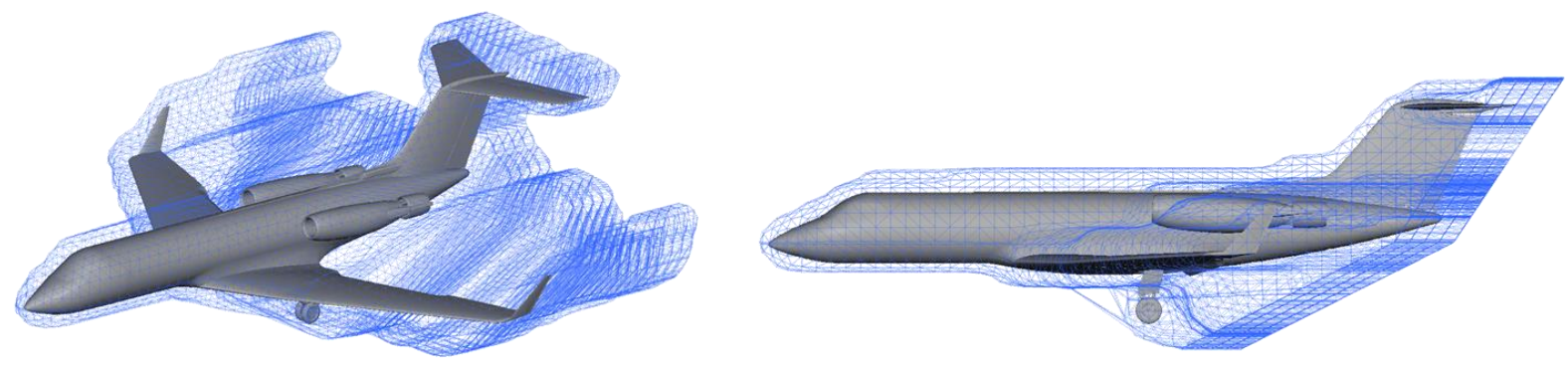

Fig. 8 Views of optimum permeable surface used during simulation as shown on the ACTE configured G-III.

Acoustic comparisons between the two measurement surfaces are shown in Fig. 9. For the purposes of this section, we are comparing the ACTE configuration with flap deflection $\delta_{\mathrm{f}}=25^{\circ}$, MLG deployed, porous fairings installed, at an $\mathrm{AOA}=4.65^{\circ}$, and in fine resolution. In the low frequencies, up to approximately $700 \mathrm{~Hz}$, there is good agreement between the two measurement surfaces. Beyond this frequency, the two datasets begin to diverge, with the solid surface data trending toward higher values. With the solid measurement surface, the volumetric effects that occur near the aircraft are not included in the acoustic results. In contrast, the permeable measurement surface is offset from the aircraft and therefore accounts for the volumetric effects. Of concern is the hydrodynamic content that passes through the aft faces of the permeable surface in the form of wake vortices/structures. The semiplanar endcaps visible in Fig. 8 are used for averaging purposes in an attempt to filter out or diminish the effects of hydrodynamic pressure fluctuations. Due to constraints on the computational cost of the simulation, the voxel size must be decreased out to the permeable measurement surface. The frequency resolution that can be resolved depends on how many voxels are captured per wavelength; thus, this reduction in spatial resolution causes an underestimation in the high frequency noise captured. These trade-offs lead to the conclusion that the correct spectrum will lie somewhere in between these two datasets.

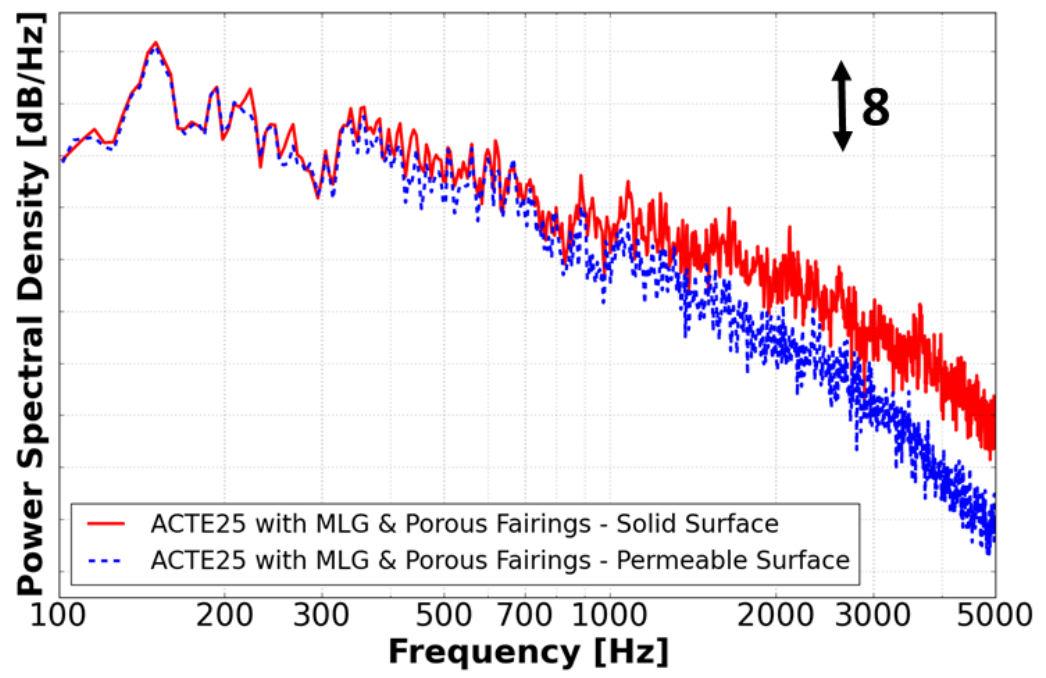

Fig. 9 Power Spectral Density (PSD) vs. frequency for ACTE $\delta_{\mathrm{f}}=25^{\circ}$ comparing solid and permeable surface results for an overhead microphone.

\section{Aerodynamic Effect - Flap Configuration}

While the main focus of this investigation was to assess the acoustic benefit of the noise reduction concepts described above, we were also keen on conducting limited aerodynamic analyses of the two flap configurations. In this section, the aerodynamic effect of each flap type is discussed for their effect on total aircraft change in lift and drag, as well as changes in flow topology in the wake of the flap. All comparisons in this section, except those in Fig. 10 , were made using full-span, medium resolution results, $\mathrm{AOA}=6^{\circ}$, and MLG retracted. The results displayed in Fig. 10 were made using half-span (with symmetry plane), coarse resolution, AOA $=6^{\circ}$, and MLG retracted. The data shown for the Fowler (baseline) flap G-III aircraft were extracted from the simulations highlighted in the companion study [3]. 

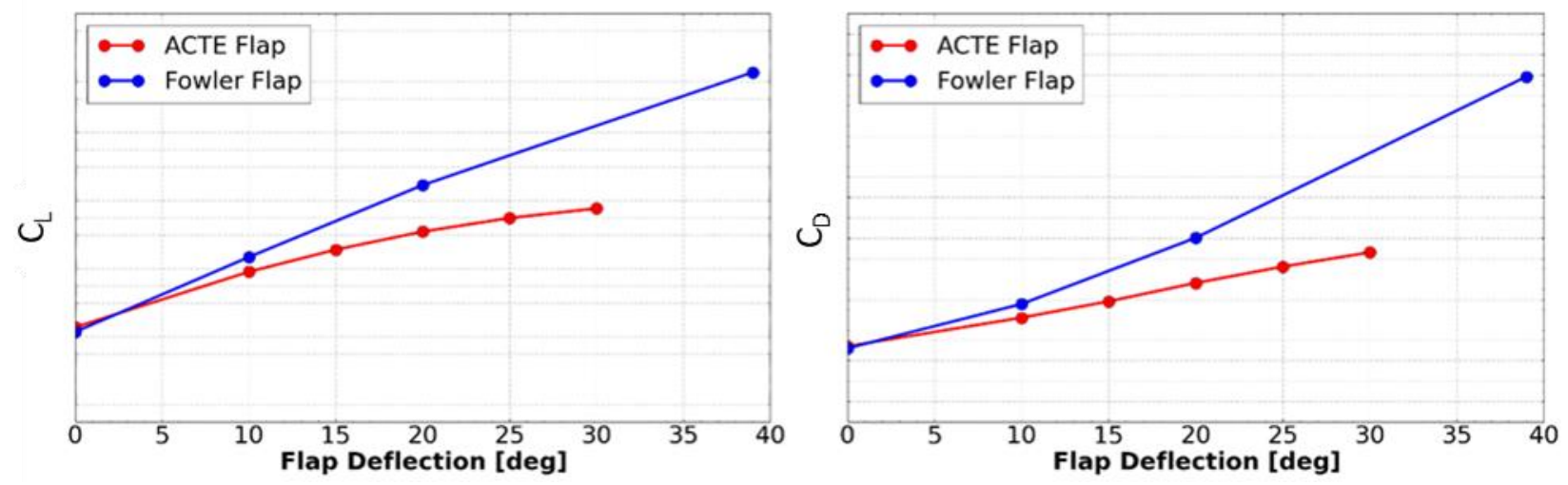

Fig. 10 Aerodynamic coefficients as a result of $\delta_{\mathrm{f}}$ at $\mathrm{AOA}=6^{\circ}$ with $\mathrm{MLG}$ retracted.

As demonstrated in previous flight tests that focused on the structural and aerodynamic aspects of the technology [9], the aerodynamic performance of the wing equipped with the ACTE flap was expected to be different from that of the baseline wing in its standard Fowler flap configuration. The difference is mainly caused by elimination of the gap at the flap leading edge. The aerodynamic coefficients, namely lift and drag, are presented in Fig. 10 for the flap deflection angles simulated. The Fowler flap configuration is able to achieve a maximum flap deflection angle of $39^{\circ}$ compared to the ACTE flap, which is limited to $30^{\circ}$. This leads to early termination of the ACTE data points when plotted against the Fowler data. The Fowler flap increase in $\mathrm{C}_{\mathrm{L}}$ of approximately $130 \%$ at $\delta_{\mathrm{f}}=39^{\circ}$ over $\delta_{\mathrm{f}}=0^{\circ}$ is in line with experimental results obtained by Paulson [28]. The ACTE realizes an increase in $\mathrm{C}_{\mathrm{L}}$ of approximately $60 \%$ at $\delta_{\mathrm{f}}=30^{\circ}$ over $\delta_{\mathrm{f}}=0^{\circ}$. The slope of the lift curve as a function of flap deflection angle shows similar trends between the two flap types, with the aircraft producing less differential lift as flap deflection increases. The increase in lift of the ACTE flap is considerably less than that of the Fowler flap, giving the former a shallower slope especially toward the maximum flap deflection angle. The Fowler flap configuration produces approximately $300 \%$ more drag at full flap deflection than without deflection, a marked increase over the ACTE flap configuration, which sees a drag increase of approximately $110 \%$ at full deflection. The slope of the drag curve as a function of flap deflection angle for the Fowler flap shows a slight increase, whereas the ACTE flap drag curve is relatively linear in slope. At lower flap deflections, the ACTE flap is thus able to achieve higher aerodynamic efficiencies. The Fowler flap configuration is able to realize much higher $C_{L}$ values at the expense of efficiency in the form of a correspondingly larger increase in $C_{D}$. Overall, the aerodynamic trends presented in Fig. 10 agree with the flight test results presented in Ref. [9]. Moreover, through discussions with the developers of the ACTE flap, we learned that aerodynamically, the ACTE flap at $25^{\circ}$ deflection was expected to perform similarly to the nominal approach condition for the Fowler flap at $20^{\circ}$ deflection. Although the predicted forces shown in Fig. 10 lend support to this notion, they suggest that the ACTE flap at $30^{\circ}$ deflection may be a slightly better match (regarding $C_{L}$ only) for the Fowler flap deflected $20^{\circ}$. Nevertheless, as part of the airframe noise flight test campaign [8], we have focused on the aeroacoustic analysis of the ACTE flap at $25^{\circ}$ with and without MLG fairings and comparisons with the baseline G-III aircraft in its approach condition of Fowler flaps set at $20^{\circ}$.

The increase in lift for the Fowler flap-equipped G-III is evident in Fig. 11, which shows streamwise $C_{P}$ distributions on the surface of the wing at the inboard (116 in), mid (201 in) and outboard (269 in) section of the flap. The red curve corresponds to the Fowler flap configuration, with separate contours for the main wing body and the flap surface, while the black curve shows data for the ACTE flap configuration. A noticeable feature of the ACTE flap curve are the oscillations in $C_{P}$ values present on both the pressure and suction sides at dimensionless values of $\mathrm{x}=0.8$ and $\mathrm{x}=0.76$, respectively. These oscillations are attributable to the presence of a narrow trough on the surface of the wing that runs along the span at the transition juncture where the ACTE flap mates with the main wing body. Due to the elimination of the leading-edge gap, the suction peak of the ACTE flap is weaker and more gradual than that of the Fowler flap, which features sharp suction peaks that taper off rapidly toward weaker pressure values. The pressure distribution on the ACTE flap is much more uniform along its span than that of the Fowler flap. This behavior is caused by the elimination of ancillary geometries (e.g., flap brackets, tracks) on the surface. The effect of these secondary components is evidenced in the midspan plot that shows oscillations of the $\mathrm{C}_{\mathrm{P}}$ values near the suction peak followed by an abrupt separation. The leading edge of the Fowler flap contains a set of turning vanes that cause the $C_{P}$ values to oscillate in that region, while the separation is a result of the $C_{P}$ cut having been taken adjacent to a flap track. The plots taken at spanwise locations of 116 inches and 269 inches are more indicative of the behavior in a 
clean region of the Fowler flap, without any obstructions on the surface. The region of separation just downstream of the suction peak on the ACTE flap is typical of this configuration and was present along the entire span. The subtle differences in the upstream effect of the two flap configurations are visible in these plots, with the Fowler flap having slightly larger suction peaks at the leading edge of the wing.
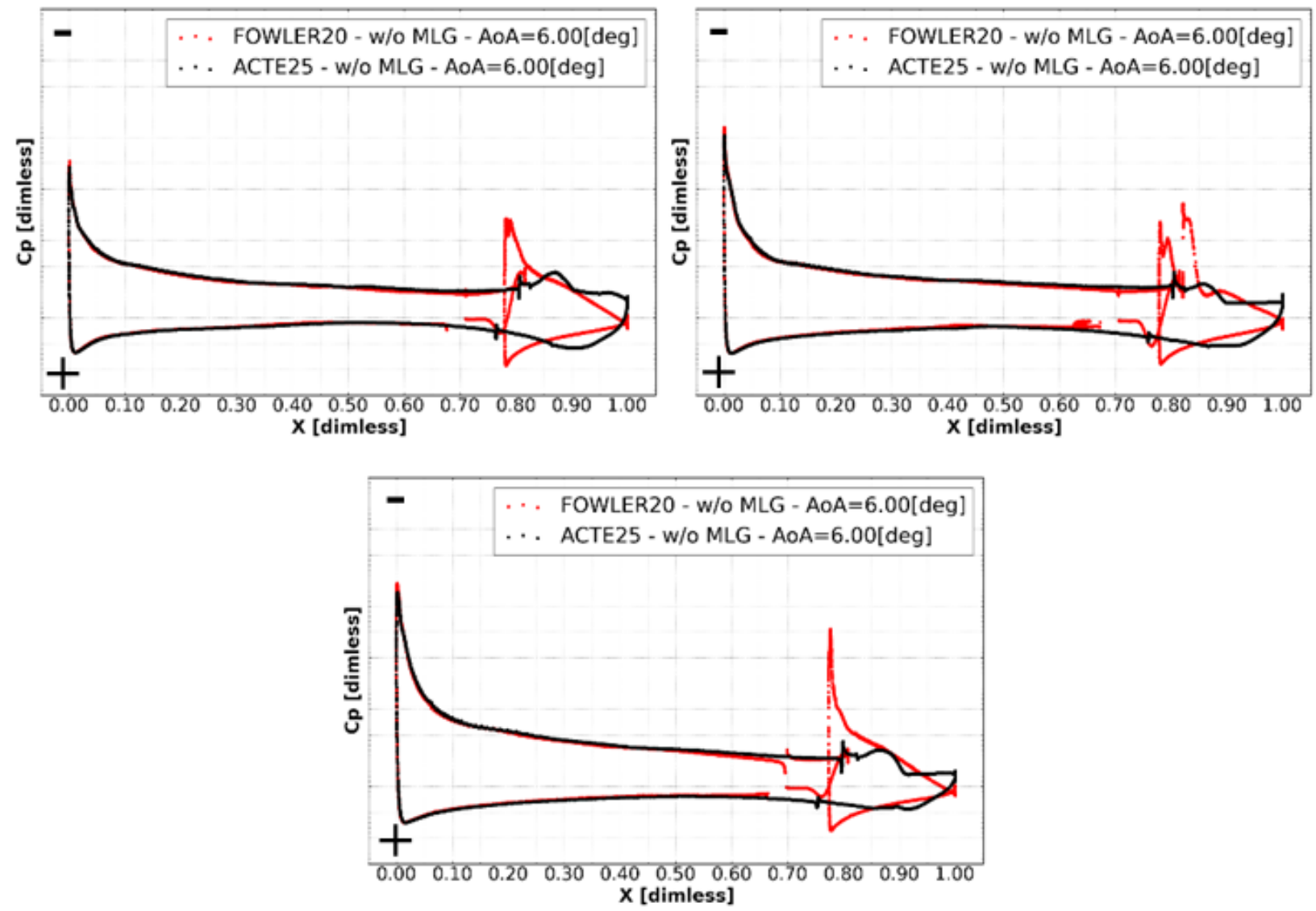

Fig. $11 \mathrm{C}_{\mathrm{P}}$ profiles comparing the ACTE $\left(\delta_{\mathrm{f}}=25^{\circ}\right)$ and Fowler $\left(\delta_{\mathrm{f}}=20^{\circ}\right)$ flap configurations. Clockwise from top left: inboard flap, mid flap, outboard flap.

A comparative look at surface pressure distributions for the Fowler and ACTE configurations at their respective $20^{\circ}$ and $25^{\circ}$ flap deflection angles is shown in Fig. 12. The low-pressure regions over the Fowler flap leading edge and on the flap side-edges are clearly visible. Also note from the figure three localized regions of separated flow on the suction side of the Fowler flap, one in-between each flap track, with the regions getting progressively smaller toward the outboard flap tip. The ACTE flap has a low-pressure bias on the suction side toward the inboard flap region, with a relatively uniform pressure distribution along the span that is finally diminished at the transition zone near the outboard flap tip. The relative lack of pressure gradient, as evidenced by the uniform coloring, on the surface of the ACTE flap indicates that the flow separation visible in Fig. 12 does indeed extend across the entire span of the flap surface. As mentioned earlier, the pressure distribution along the leading edge and forward portion of the wing shows no substantial change between the Fowler and ACTE flap configurations. Finally, we note that the slit in the ACTE flap configuration, at the juncture between the flap and main wing body on the inboard side-edge, promotes seepage (or jetting) of the fluid from the wing pressure side to the suction side that causes the appearance of a narrow streamwise low pressure zone.

A final look at the aerodynamic behavior of these two configurations is presented in Fig. 13, which shows flownormal slices that were extracted downstream of the flap and represent contours of velocity magnitude. The slice taken in the wake of the Fowler flap shows typical flow behavior for this configuration, that is, the presence of flap side-edge vortices and regions of low-speed flow downstream from the two flap middle tracks. The slice taken in the wake of the ACTE flap shows faint evidence of flap vortices caused by wake roll-up at the inboard and outboard 
transition segments and a large region of relatively low-speed flow that spans the entire flap. Note that the flap sideedge vortices emanating from the ACTE flap are much weaker than those from the Fowler flap.

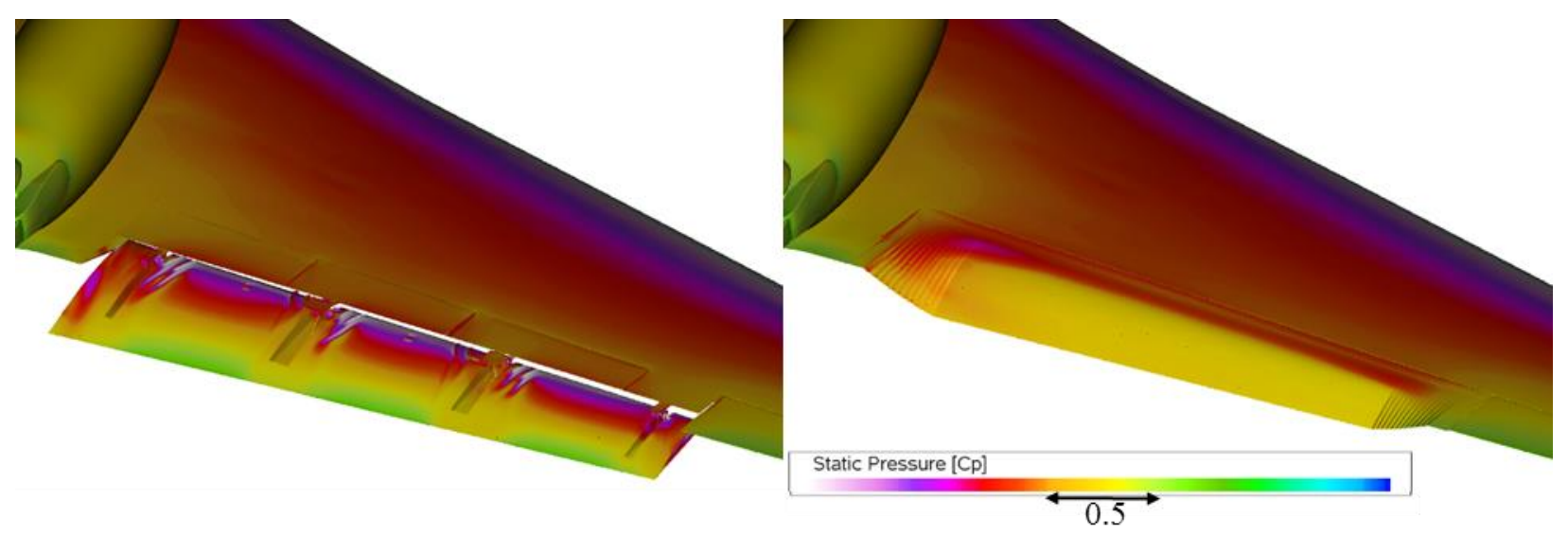

Fig. 12 Time-averaged pressure distributions on the upper surface of the wing for the Fowler $\left(\delta_{\mathrm{f}}=20^{\circ}\right.$, left) and $\mathrm{ACTE}\left(\delta_{\mathrm{f}}=25^{\circ}\right.$, right $)$ configurations.

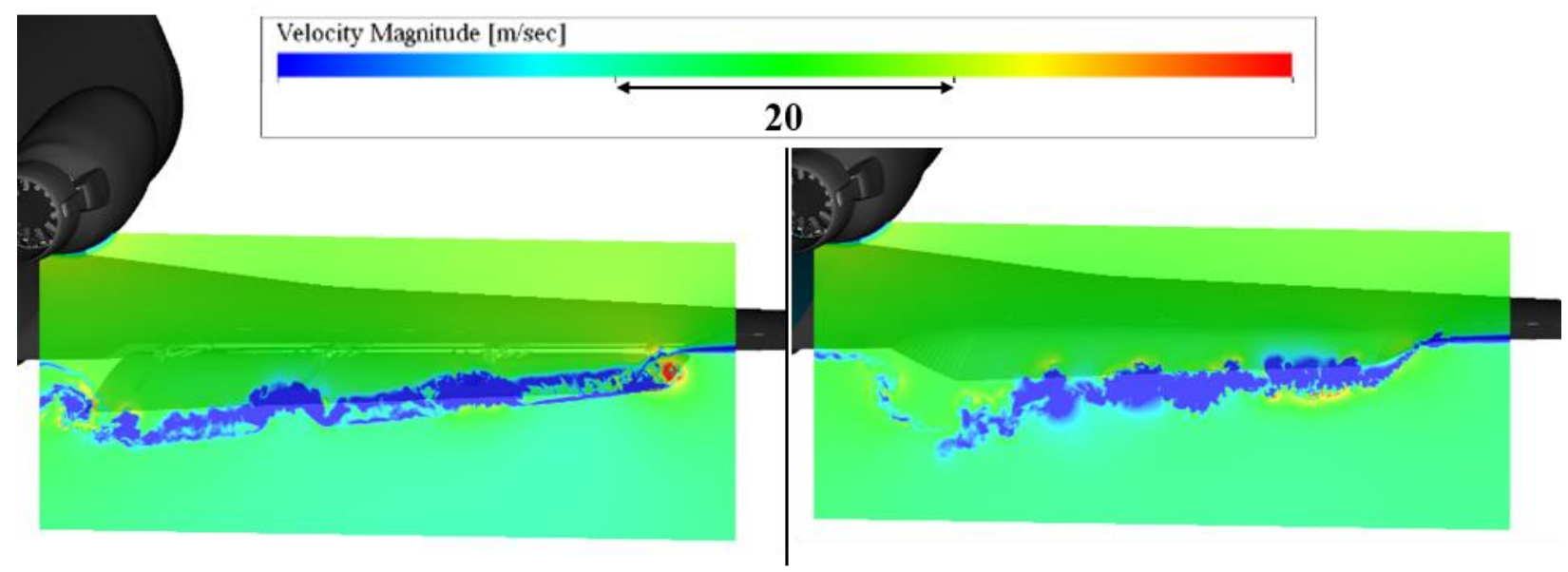

Fig. 13 Instantaneous velocity magnitude on flow-normal planes downstream of the wing for the Fowler $\left(\delta_{\mathrm{f}}=20^{\circ}\right.$, Left $)$ and $\mathrm{ACTE}\left(\delta_{\mathrm{f}}=25^{\circ}\right.$, Right $)$ flap configurations.

\section{Aeroacoustic Effect - Flap Configuration}

The acoustic performance of the ACTE technology on a component-level basis, without the effects of landing gear, is examined in this section. Data presented here were extracted from medium resolution simulations of the fullspan aircraft at $\mathrm{AOA}=6^{\circ}$ and compare the aerodynamically equivalent deflection angles of $20^{\circ}$ for Fowler and $25^{\circ}$ for ACTE flap configurations. Also included in the comparison are acoustic results for the ACTE flap at deflection angle of $0^{\circ}$. While small differences exist between the G-III model with the ACTE flap and Fowler flap at $0^{\circ}$ deflection, we will consider ACTE acoustic results to be representative of both configurations at this $0^{\circ}$ deflection angle. The ACTE flap was found to provide a substantial reduction in the overall airframe noise when compared to the traditional Fowler flap configuration when deflected.

The PNL of the various flap configurations was evaluated in accordance with the procedure outlined in Section III G. The levels, as function of time, are presented in Fig. 14 for the case in which the aircraft with the flap configurations indicated flies over an observer. The ACTE $25^{\circ}$ flap is represented by the blue line, Fowler $20^{\circ}$ flap by the red line, and the ACTE $0^{\circ}$ flap by the black line. Overall, the increase in EPNL as a result of Fowler flap deflection compared to the G-III with no flap deflected was found to be $19.1 \mathrm{~dB}$. For the ACTE flap, the same metric was $5.3 \mathrm{~dB}-\mathrm{a}$ reduction of $13.8 \mathrm{~dB}$ relative to the $20^{\circ}$ Fowler flap configuration. The magnitude of the increase in EPNL that results from deflection of the Fowler flap is evidence of the large effect that this component has on total airframe noise. From these comparisons, we can confidently state that use of ACTE technology virtually eliminates the noise associated 
with conventional Fowler flaps, a fact that is corroborated by the flight test results of Ref. [8]. However, the presence of background noise and various other secondary noise sources on the G-III aircraft precluded realization of this level of noise reduction during the flight tests of the ACTE flap [8]. Also note from Fig. 14 that the ACTE and Fowler flap configurations have similar trends as the aircraft approaches. After the aircraft passes overhead at approximately 12.5 $\mathrm{s}$, the ACTE flap indicates a more rapid reduction in noise level as compared to the Fowler flap configuration. The downward trend of the ACTE curve continues to the end of the sampled period, whereas the Fowler flap curve shows a shallower slope.

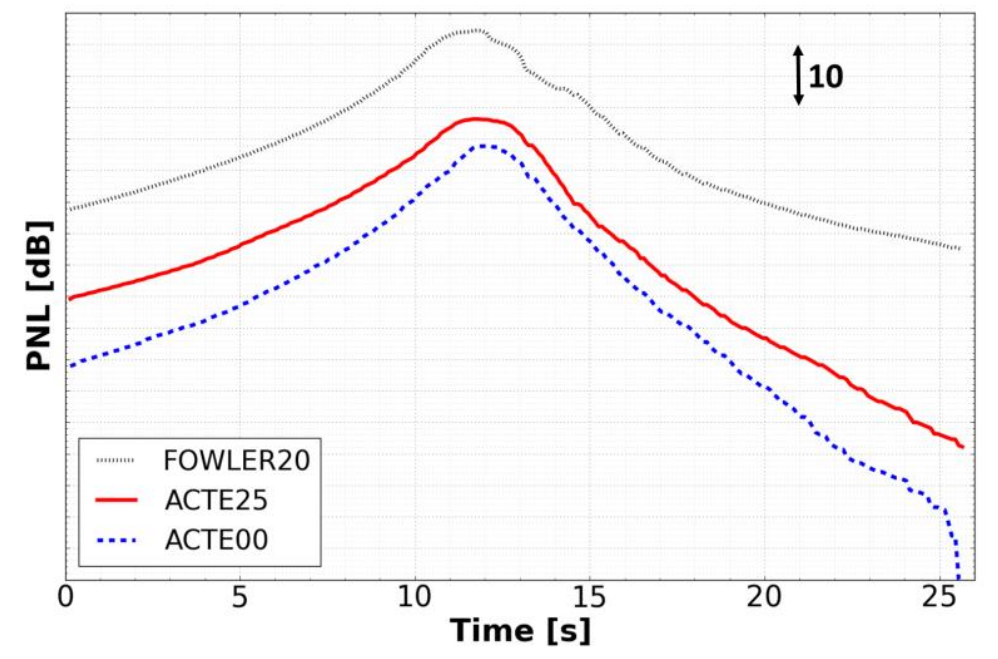

Fig. 14 PNL vs Time.

The acoustic directivity was analyzed on a $120 \mathrm{~m}$-radius semicircle along the longitudinal mid-plane of the aircraft. Results from the simulations were extracted every half degree on the semicircle. Figure 15 shows SPL contours generated for the ACTE configuration at $0^{\circ}$ deflection, ACTE configuration at $25^{\circ}$ deflection, and Fowler configuration at $20^{\circ}$ deflection. All three plots indicate, across the frequency spectrum and to varying levels, a bias in the forward directivity ranging from $0^{\circ}$ to $90^{\circ}$. This is especially evident in the deflected Fowler flap case, with a large area of high noise content present between $0^{\circ}$ to $60^{\circ}$ that is essentially broadband in nature, ranging from 300 to $4000 \mathrm{~Hz}$. The deflected ACTE flap case shown is much quieter than the deflected Fowler flap case, but, as expected, noisier than the undeflected ACTE as evidenced by higher levels in the mid-frequency range, 500 to $2000 \mathrm{~Hz}$, that extend further aft in the directivity range. Additionally, with the ACTE flap deflected, there is indication of elevated noise levels throughout the forward directivity range below $150 \mathrm{~Hz}$.

The corresponding spectral plots for the same three configurations are presented in Figure 16. The spectra, which are provided for $60^{\circ}, 75^{\circ}, 90^{\circ}$, and $105^{\circ}$ directivity angles, give a more detailed look at the frequency content and levels than what can be gleaned visually from the contour maps depicted in Figure 10. The ACTE flap seems to be as noisy as the baseline Fowler flap for frequencies below $100 \mathrm{~Hz}$, likely a result of the large-scale flow separation along the span of the flap. However, the simulated record length does not provide enough low frequency content to make a definitive statement regarding spectral behavior below $100 \mathrm{~Hz}$. For frequencies above $100 \mathrm{~Hz}$, the Fowler flap produces considerably more noise than the ACTE flap, especially in the mid-to-high frequency ranges. This is a result of the strong flow unsteadiness produced by the flap side-edges and bracket assemblies. With no flap deflected, there appears to be some tonal content being generated between 1500 and $2200 \mathrm{~Hz}$. This is most evident in the mid-toforward directivities, with signs of diminishing strength as the observer moves aft of aircraft center. 

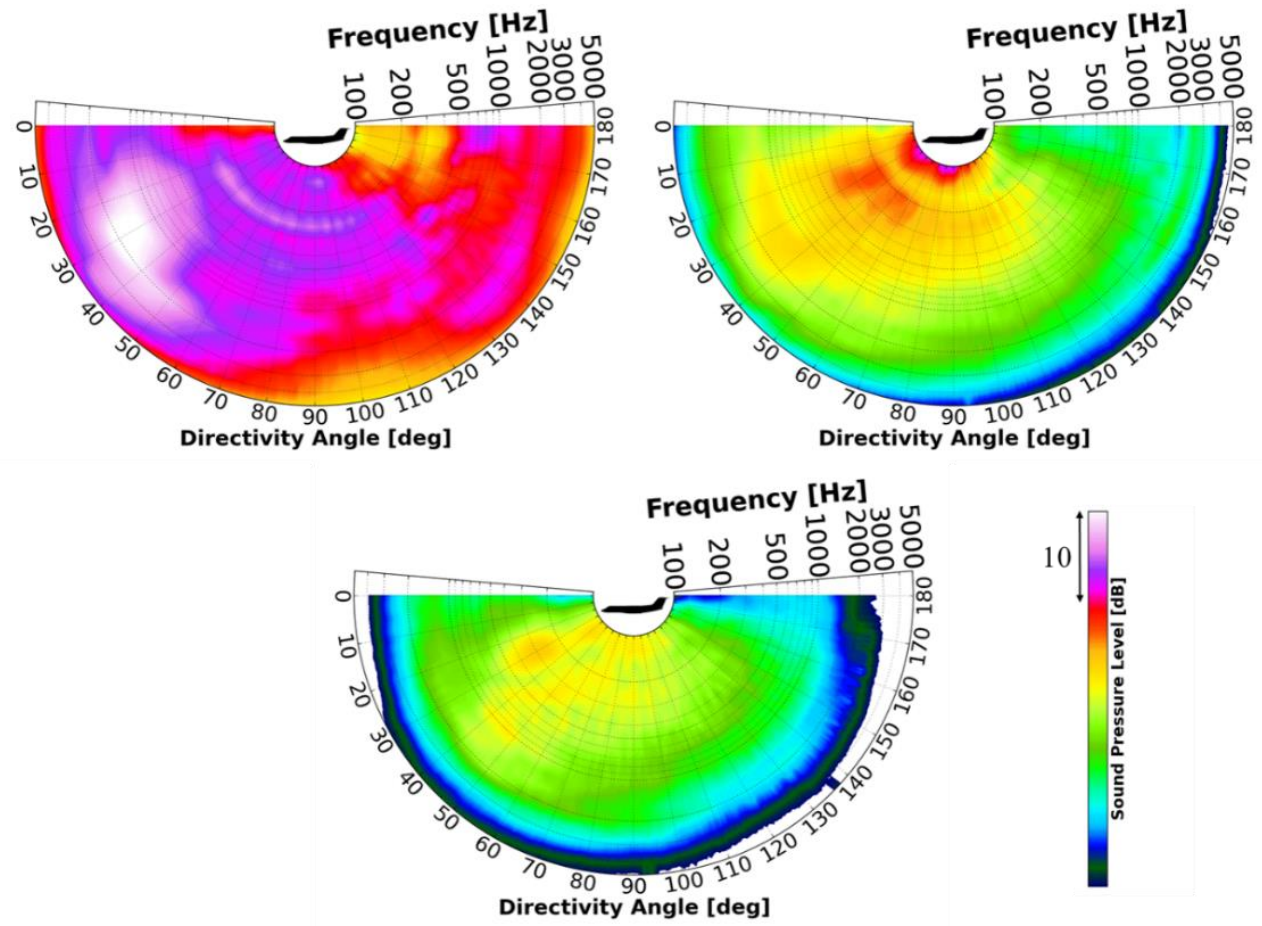

Fig. 15 Directivity contour maps in $1 / 3^{\text {rd }}$ octave bands. Fowler flap with $\delta \mathrm{f}=20^{\circ}$ (upper, left) and ACTE flap with $\delta_{\mathrm{f}}=25^{\circ}$ (upper, right), and G-III without flap deflection (lower).
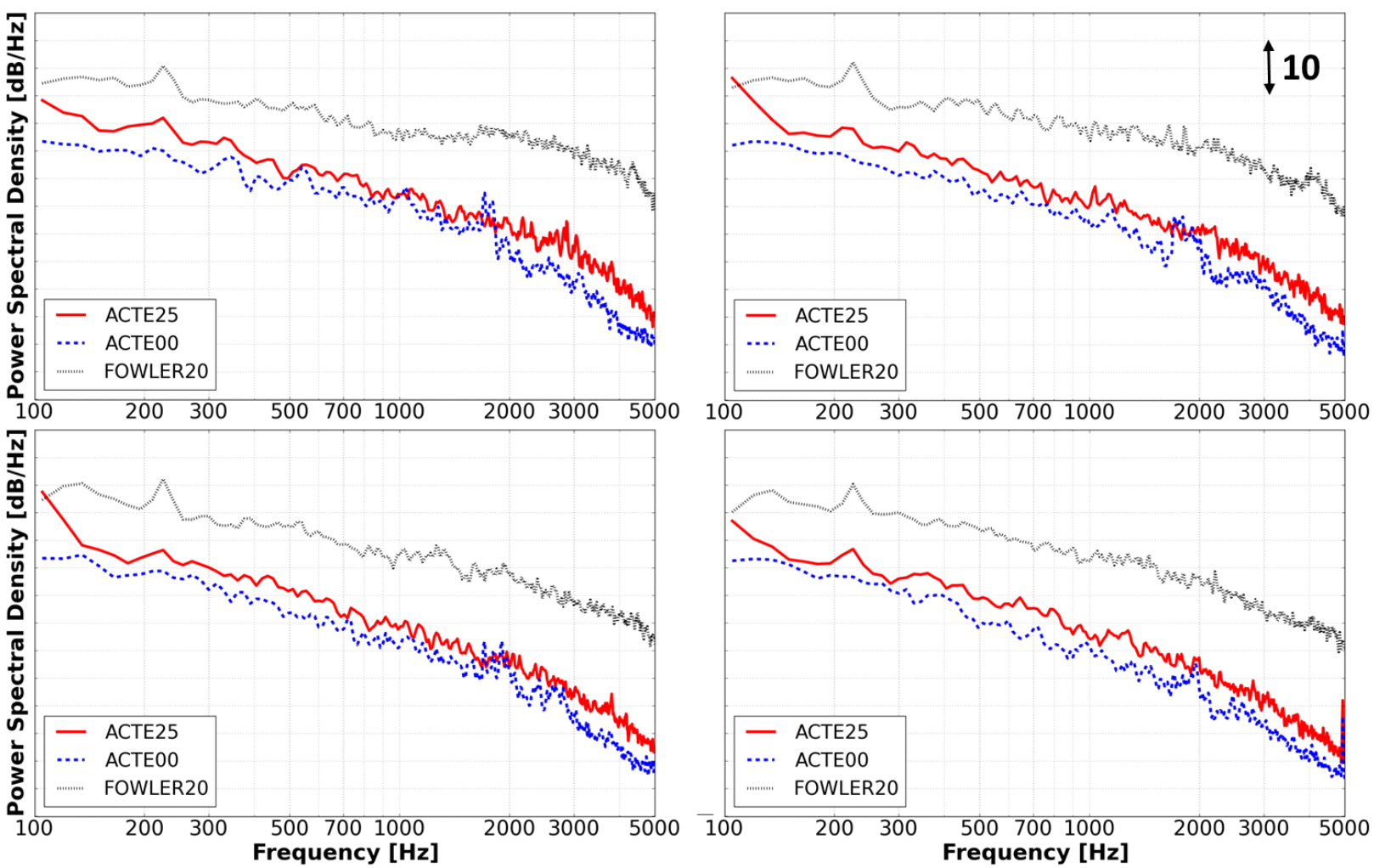

Fig. 16 Power Spectral Density (PSD) vs. frequency for ACTE $\delta_{\mathrm{f}}=0^{\circ}$ (black), ACTE $\delta_{\mathrm{f}}=25^{\circ}$ (blue), and Fowler $\delta_{\mathrm{f}}=20^{\circ}$ (red). $60^{\circ}$ directivity (upper, left). $75^{\circ}$ directivity (upper, right). $90^{\circ}$ directivity (lower, left). $105^{\circ}$ directivity (lower, right). 


\section{E. Aeroacoustic Effect - MLG Deployment}

A brief examination of acoustic changes related to landing gear deployment on the ACTE configured aircraft with a flap deflection $\delta_{\mathrm{f}}=25^{\circ}$ is presented here. Data correspond to full-span, medium-resolution simulations with $\mathrm{AOA}=4.65^{\circ}$. All flow parameters were consistent between simulations. This section is meant to familiarize the reader with the effect of the unfaired MLG before and after deployment before investigating the effects of applying the porous knee fairings to an already deployed MLG in Section F.

The impact of MLG deployment on EPNL can be inferred from Fig. 17. In terms of comparative values, the deployment of the main landing gear causes an increase in EPNL of $15.1 \mathrm{~dB}$ and a corresponding increase in $\mathrm{PNL}_{\max }$ of $14.4 \mathrm{~dB}$. With the understanding that one of the largest contributors to airframe noise is the presence of strong flow separation, the relatively large increase in noise associated with the deployment of the landing gear is not surprising. The ACTE-equipped G-III aircraft, with MLG retracted, produces very little flow separation/unsteadiness and correspondingly not much noise. Therefore, the deployed MLG becomes the dominant airframe noise source on the aircraft. Indeed, if one were to compare with the EPNL data discussed in Section D, the inference could be made that the ACTE-equipped, MLG-deployed G-III configuration produces similar noise levels to those of the Fowler-equipped G-III with MLG retracted. This is an indication that, on a G-III aircraft, the MLG and Fowler flaps are comparable noise sources. With the elimination of flap noise as a dominant component, deciphering the full extent of the noise signature associated with the main landing gear is a less challenging task.

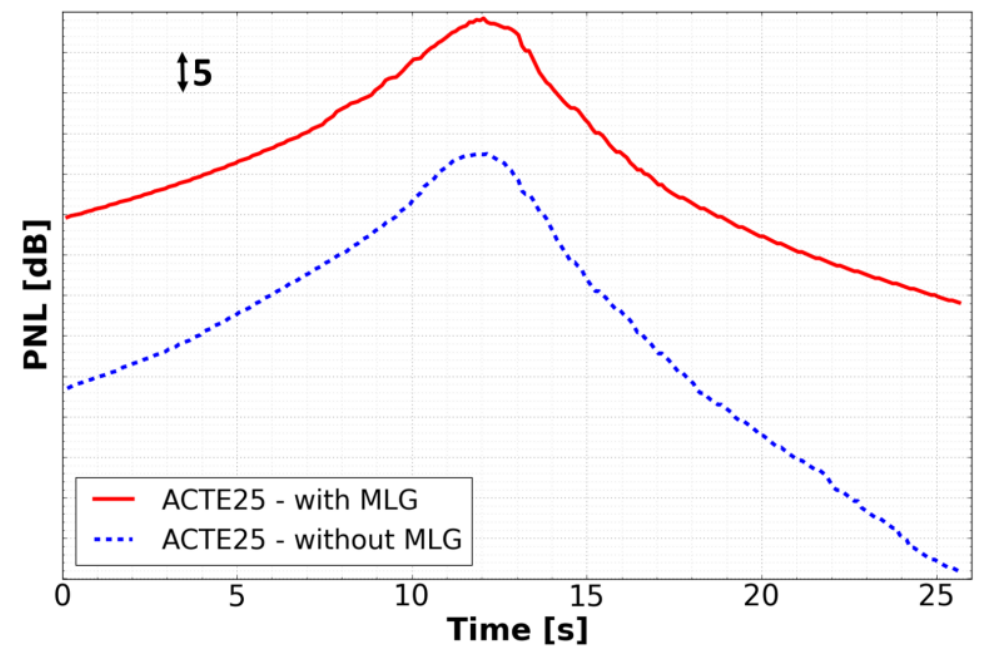

Fig. 17 PNL vs time showing the effect of MLG deployment.

\section{F. Aeroacoustic Effect - Porous Fairings}

This section presents an examination of acoustic changes related to application of the porous fairings on the ACTEconfigured aircraft at an $\mathrm{AOA}=4.65^{\circ}$ with a flap deflection $\delta_{\mathrm{f}}=25^{\circ}$. Data were extracted from full-span, fineresolution simulations with all flow parameters consistent between simulations. Where comparable data existed, results from the Fowler flap configured aircraft with flap deflection $\delta_{\mathrm{f}}=20^{\circ}$ are also included.

PNL as a function of time is shown in Figure 18. The plot demonstrates the effect of the porous knee fairings when applied to the ACTE configured aircraft with a flap deflection $\delta_{\mathrm{f}}=25^{\circ}$. Overall, the results show EPNL is reduced by $3.31 \mathrm{~dB}$ while $\mathrm{PNL}_{\mathrm{MAX}}$ is reduced by $3.34 \mathrm{~dB}$. As the aircraft passes overhead, with the line of sight from the aircraft to the observer moving from the front side of the MLG to the rear, the magnitude of the reduction in PNL becomes comparably less. Since EPNL is used by the FAA for aircraft certification standards, the reduction in this value shown here is a positive result for the set of porous knee fairings as a viable noise reduction concept. 


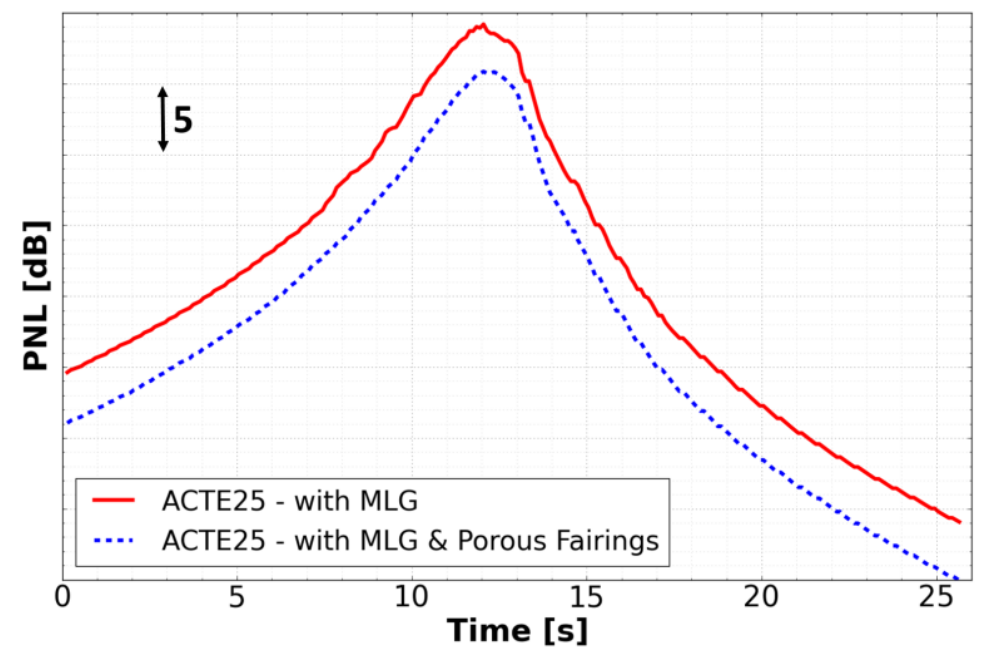

Fig. 18 PNL vs time showing the effect of installing the porous knee fairings.

Directivity contour maps for the ACTE configured G-III with $\delta_{\mathrm{f}}=25^{\circ}$ are shown in Fig. 19. These images compare the simulation data without porous knee fairings to those data with the noise reduction concept installed. The fairings are shown to produce a marked reduction in both broadband and tonal responses. To aid in the analysis, we will divide the frequency ranges into three categories: low frequency below $500 \mathrm{~Hz}$, mid-frequency between $500-2000 \mathrm{~Hz}$, and high frequency above $2000 \mathrm{~Hz}$. The reductions in the mid-to-high frequency ranges are visible across all directivities. The MLG structure is comprised of numerous bluff bodies of different sizes and shapes which, due to their various flow separation patterns, generate noise across a wide range of frequencies. By shielding these bodies, and thus either halting or diminishing flow unsteadiness, a reduction or elimination in noise is achieved. In the initial portion of the mid-frequency range, approximately $570 \mathrm{~Hz}$, a band of high activity is visible. This will be examined in greater detail in Section $\mathrm{E}$ but is shown to be eliminated after application of the porous knee fairings. In the low-frequency range, a broadband reduction in SPL levels was also achieved, though some isolated regions of increased activity in the forward directivities remain. It was determined that the MLG bay cavity is dominant in this range and so the reduction here is attributable to aerodynamic and, consequentially, aeroacoustic differences related to this geometric feature. In the unfaired case, the unstable shear layer is able to enter the cavity and drive a resonant response. The presence of the fairing significantly changes the aerodynamic nature of the grazing flow over the cavity inlet, inhibiting the recirculation of this unsteady shear layer in the cavity, in turn reducing the resonant effect. The reduction of these acoustic waves through use of the porous knee fairings also, in small part, ensures the energy entering the bay cavity is correspondingly reduced. Similar to the region of high activity around $570 \mathrm{~Hz}$, the MLG bay cavity response will be explored in greater detail in Section E.
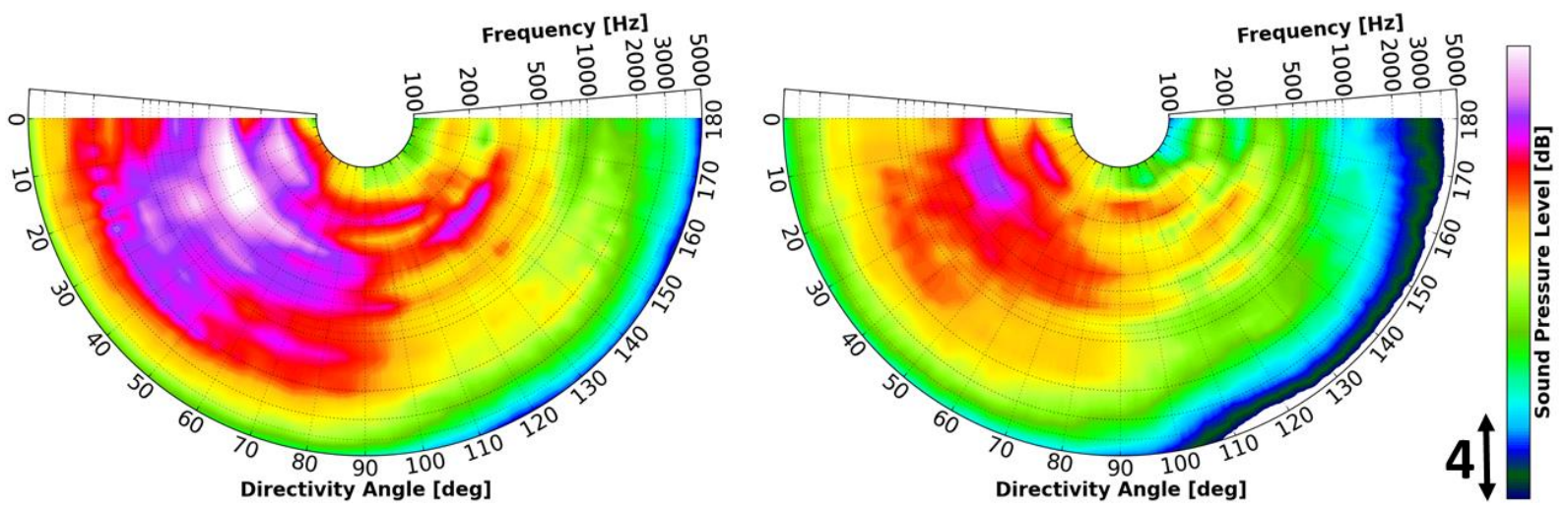

Fig. 19 Directivity contour maps in $1 / 3^{\text {rd }}$ octave bands from the GIII ACTE with $\delta \mathrm{f}=25^{\circ}$. Untreated MLG, no porous knee fairings (left) and treated MLG, with porous knee fairings (right). 
Figure 20 compares power spectral density (PSD) plots with and without MLG porous knee fairings. For comparison, also included is the spectrum for the G-III with the Fowler flap system at a flap deflection $\delta_{\mathrm{f}}=20^{\circ}$. Reviewing the spectra in this manner gives greater insight into their behavior at a given directivity. In each plot, the Fowler flap configuration possesses higher sound pressure levels than the ACTE flap configuration, most notably in the high frequency range at the $60^{\circ}$ directivity. Comparing the unfaired result to that of the faired result, we can see the reduction is indeed broadband in nature, regardless of directivity. There are notable features present in the unfaired spectra, some tones that are not present in the case with porous knee fairings. These will be examined in the next section.
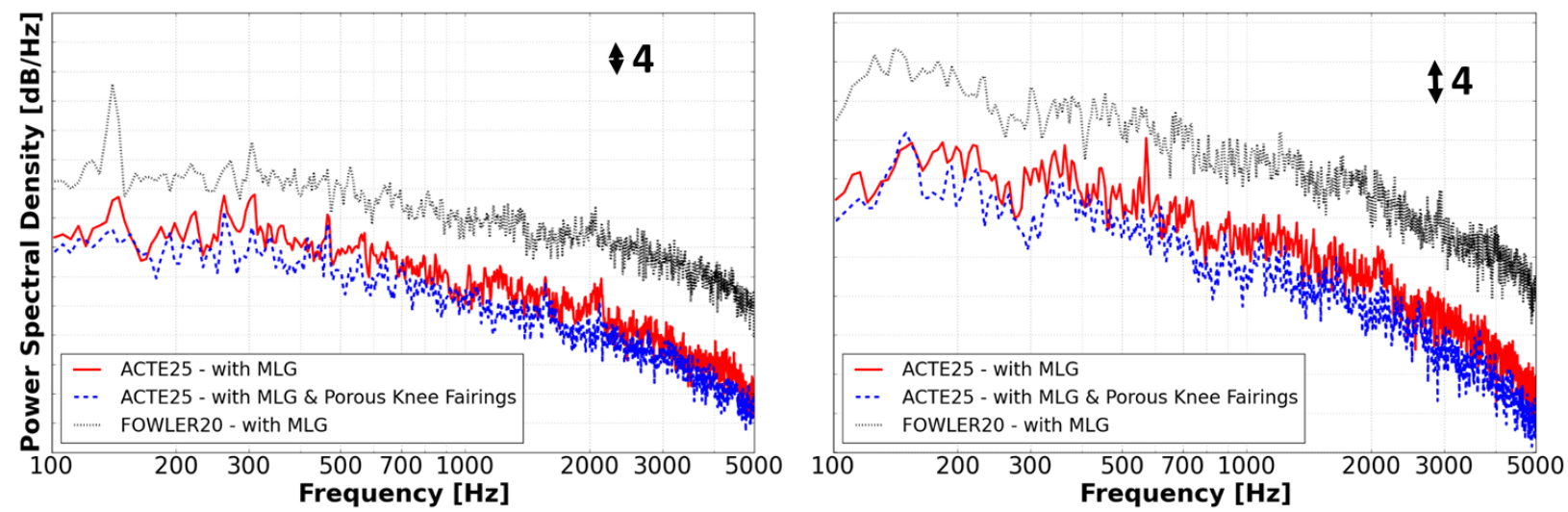

Fig. 20 Power Spectral Density (PSD) vs Frequency at $60^{\circ}$ (left image) and $90^{\circ}$ (right image) directivities.

\section{G. Tonal Investigations}

The farfield noise spectrum for the baseline gear without porous knee fairings for a single microphone, representing the $90^{\circ}$ directivity, is presented in Fig. 21 for $\mathrm{ACTE} \delta_{\mathrm{f}}=25^{\circ}, \mathrm{AOA}=4.65^{\circ}$. From this spectrum, obtained from fine resolution results, four tones were identified and selected for further investigation. These are centered on $175 \mathrm{~Hz}, 350$ $\mathrm{Hz}, 570 \mathrm{~Hz}$, and $2075 \mathrm{~Hz}$, respectively. In order to determine the components having the largest tonal contribution, a number of investigative tools were used. To quantitatively identify the largest contributors, the total SPL contribution in a given frequency range was analyzed on a part-by-part basis. From this information, a host of qualitative analyses can be made to highlight the mechanism by which the tones were being created. These analyses range from plotting the fluctuating pressures (SPL) on the surface of the aircraft to observing off-surface transient flow behaviors.

Although not shown here, subcomponent SPL contributions for the $175 \mathrm{~Hz}$ band indicated that the MLG bay cavity, pictured in Fig. 4, is the largest contributor to the overall noise level. The next largest contributor is the underside of the wing, being $4 \mathrm{~dB}$ lower than the MLG bay cavity, likely representing the footprint of the MLG bay acoustic waves propagating out of the cavity. The surface pressure map depicted in the left image of Fig. 22 indicates the presence of an acoustic wave inside the middle of the cavity. The mechanism by which this $175 \mathrm{~Hz}$ tone is generated is similar to a grazing flow, as the freestream traverses from the forward section of the wing, across the MLG bay opening and then towards the aft section of the wing. The right image in Fig. 22 represents this flow behavior as the shear layer departs the leading edge of the bay opening and convects toward the rear lip. A curvature in the shear layer causes the flow to impinge on the rear bay wall. The shedding pattern of the shear layer, along with the impingement inside of opening, contribute to excite a cavity mode and causes the increase in levels within this frequency range. The tone centered on $350 \mathrm{~Hz}$ has also been shown to be generated by the MLG bay cavity, as it is a harmonic of the tone at $175 \mathrm{~Hz}$. 


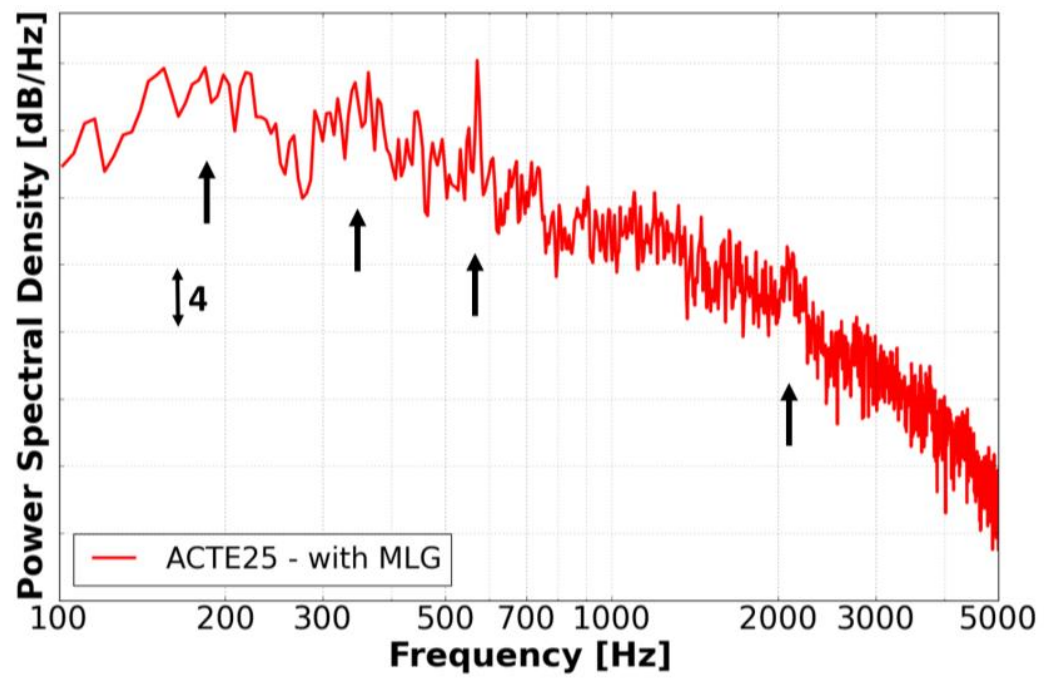

Fig. 21 Power Spectral Density (PSD) vs Frequency at $90^{\circ}$ directivity.

Similarly, reviewing the SPL contribution on a per-part basis for the $570 \mathrm{~Hz}$ tone shows the MLG forward struts as the prominent noise-producing geometric features. In Fig. 23, the cavity mode's wave pattern is clearly visible represented by the surface SPL map in the left side image. By taking the instantaneous change in pressure as a function of time on a span-normal slice intersecting the centerline of the starboard-side MLG, shown in the right side of Fig. 23, we are able to gain some clarity on how the flow inside this cavity behaves. Although not very visible, the shear layer off of the forward lip of the strut gets drawn into the strut cavity and deflects upward, impacting the back wall. The shear layer impingement zone is evidenced by a high-pressure region marked by the red and purple color contours in Fig. 23. Just above this region, striated bands of high and low pressure, which alternate in time, produce the strong tone centered at $570 \mathrm{~Hz}$. The method by which the physical G-III aircraft was converted into a digital model, described in Section II, was not able to fully resolve the internal components of this cavity. A decision was made early on in the study to use the outer mold line of the forward strut and apply some internal thickness to capture a representation of the cavity. As a result, the simulated tonal frequency/strength, while being accurate to the modeled geometry, does not directly correspond to the values obtained from flight test measurements [8].

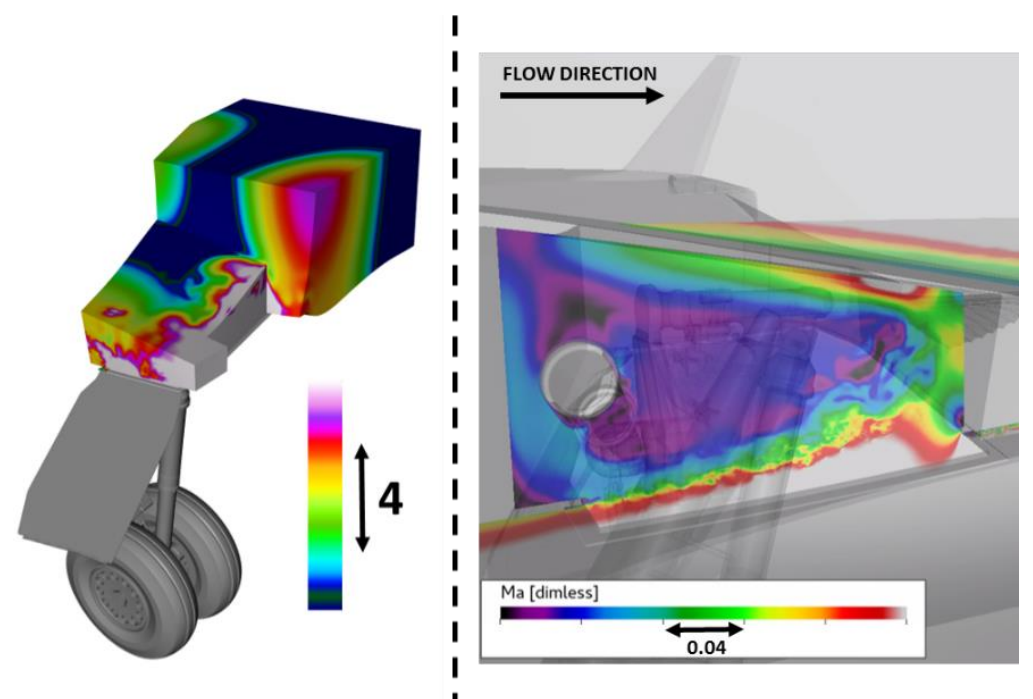

Fig. 22 ACTE $\delta_{\mathrm{f}}=25^{\circ}$ with MLG, fine resolution. Left image: Surface dB map plotted on the port-side MLG bay cavity in the 170-180 Hz band. Right image: planar streamwise flow near the inner edge of the MLG bay cavity opening. 


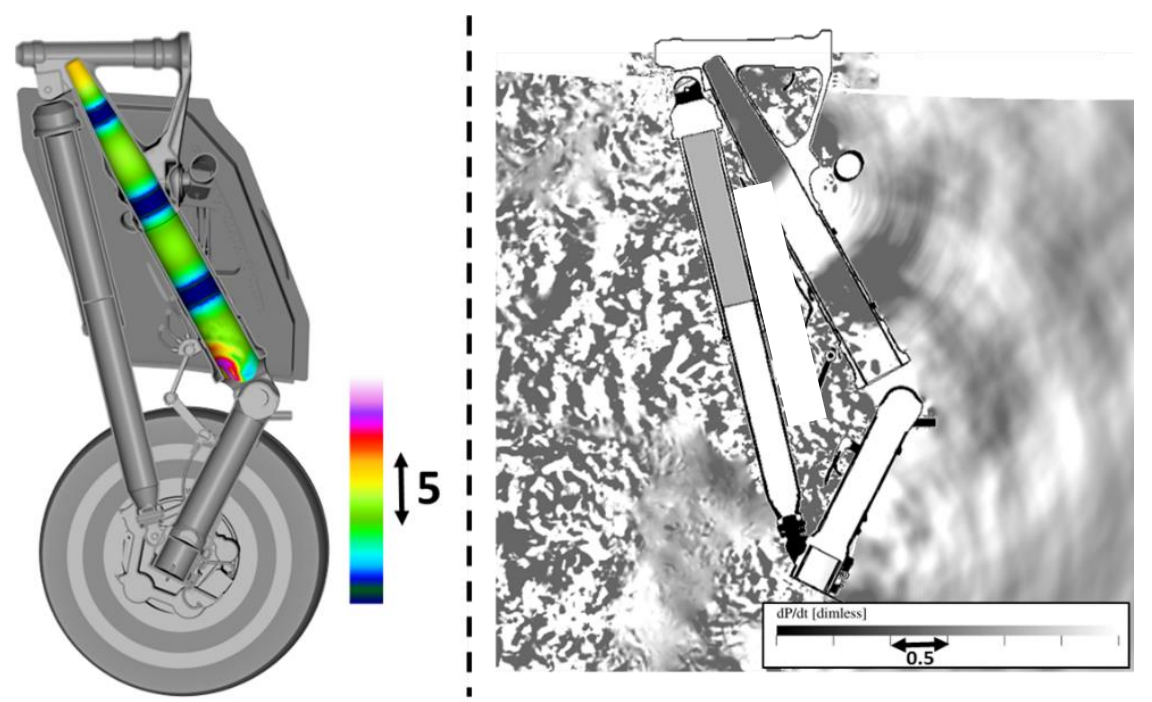

Fig. 23 ACTE $\delta=25^{\circ}$ with MLG, fine resolution. Left image: Surface $\mathrm{dB}$ map inside of the forward strut in the 560-580[Hz] band. Right image: $\mathrm{dP} / \mathrm{dt}$ on a plane intersecting the center of the MLG, with the MLG structure outlined.

The tone centered around $2075 \mathrm{~Hz}$ was shown to be emanating from the hoses that are located along the loadbearing MLG structures. The surface dB map shown in Fig. 24, upper image, represents the localized spots where increased-amplitude surface pressure fluctuations are present. Specifically, two locations are identified with one being just above and aft of the knee joint and the other being toward the top of the forward strut. The mechanism behind this tone, unlike the cavity mode excitement of the previous tones, is a direct result of vortex shedding produced by the hoses. The lower images in Fig. 24 show instantaneous velocity on a horizontal plane intersecting the hoses within the regions of interest. In both areas and for differing reasons, an asymmetry exists that causes accelerated flow on the side where the hoses are positioned. With respect to the hoses around the knee joint, the existence of the squat sensor on the outside of the knee joint causes a localized acceleration of the flow up and around the forward strut. This accelerated flow impinges on the hoses behind the knee joint and generates shedding at the $2075 \mathrm{~Hz}$ frequency. The hoses on the upper portion of the forward strut are within what appears to be the shear layer detached from the larger strut member, causing the excitation on these hoses that is being perceived as a tone. The sidestay arm, positioned just forward of these hoses on the upper strut, causes a region of low-speed flow in its wake that limits the upper extent of the excitation.

The tonal features of the G-III MLG components without the porous MLG knee fairings, as realized on a microphone at a $90^{\circ}$ directivity, have been identified and explained. The signature of the cavity tones emanating from the MLG bay and forward strut are present in the simulation results due, in part, to the geometric concessions made while computationally modeling the physical aircraft. The bay cavity tone would be altered by a more detailed representation of the internal componentry present in the physical aircraft. Similarly, the comparability of the forward strut tone to physical flight test results would likely benefit from a more accurate representation of the volume present in the strut interior. Finally, we must note that the tones identified with the hoses are from structures that were represented as rigid in the simulations. Moreover, the position and orientations of these hoses were obtained from laser scans of the MLG with the aircraft on the ground. In reality, some of the hoses are flexible and their orientations and positions may be altered by the airflow during flight. As such, they may produce a different tonal behavior in the measured data from flight test. 


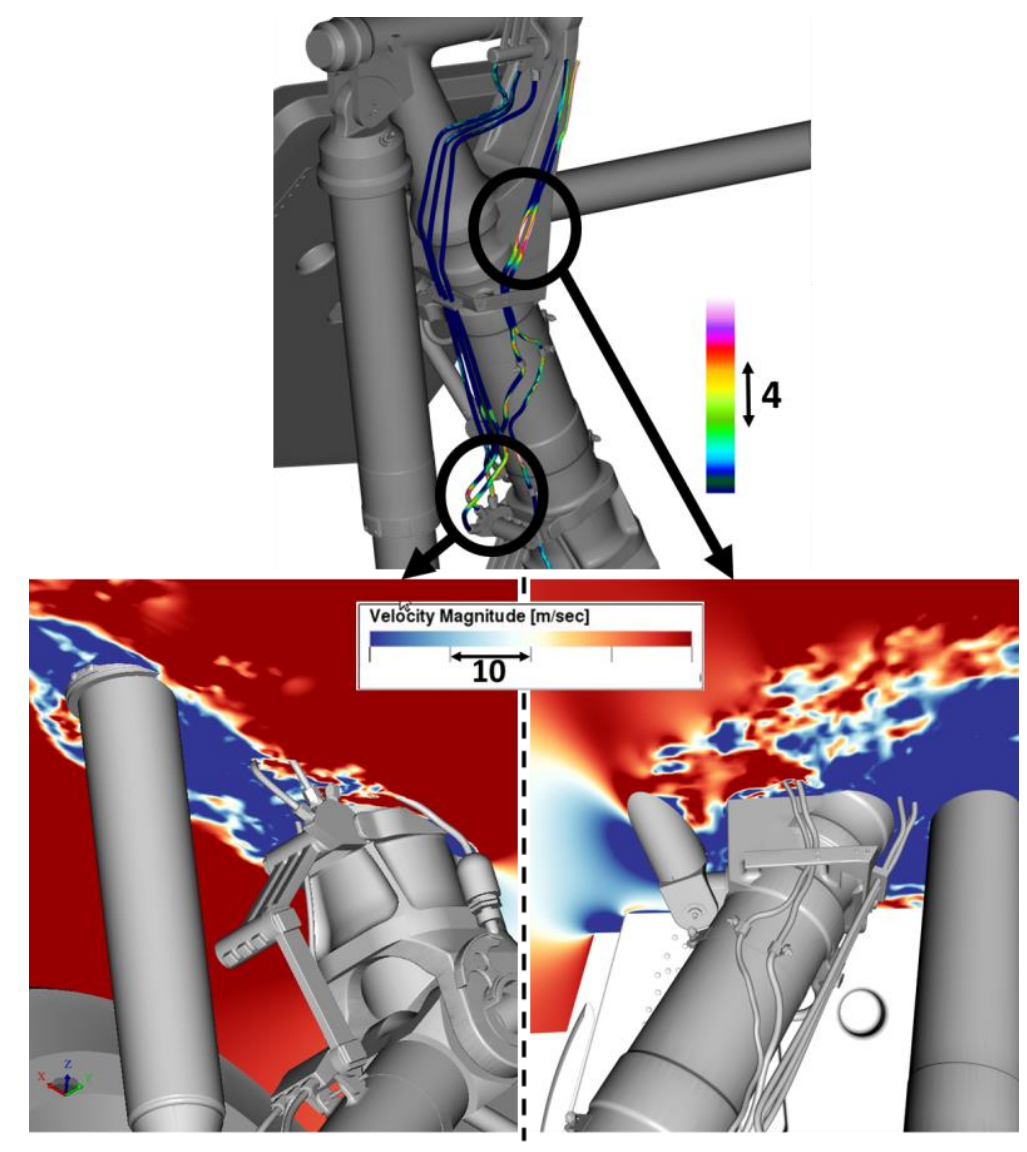

Fig. 24 ACTE $\delta_{\mathrm{f}}=\mathbf{2 5 ^ { \circ }}$ with MLG, fine resolution. Upper image: Surface dB map on hoses around the MLG structure in the 2050-2100 Hz band. Lower left image: Velocity magnitude on a horizontal plane near the MLG knee joint. Lower right image: Velocity magnitude on a horizontal plane near the upper forward strut.

\section{Concluding Remarks}

Detailed aeroacoustic analyses of the full-scale G-III aircraft with ACTE flaps without and with MLG noise reduction fairings were undertaken and summarized in this paper. The analyses were based on large-scale simulations of a high-fidelity aircraft model. Component-level aeroacoustic performance of the ACTE technology was determined for an aircraft configuration with the MLG retracted. The clean design of the ACTE flap system, without the need for exposed tracks and side-edges, greatly reduced the noise-producing flow separation zones seen on the Fowler flap equipped aircraft. The two flap systems had markedly different aerodynamic performances, with the ACTE flap system producing less lift and drag compared to the Fowler flap system for similar deflection angles. At $25^{\circ}$ deflection, the aerodynamic performance of the ACTE flap is equivalent to that of the baseline Fowler flaps deployed at $20^{\circ}$.

With the MLG stowed, use of the ACTE flap leads to a drop in EPNL of $13.8 \mathrm{~dB}$, thus virtually eliminating the noise associated with the aircraft flaps. Elimination of the flap noise enabled the accurate prediction of noise reduction benefits associated with a set of MLG fairings. Application of the porous knee fairings to the ACTE flap configured G-III provided further EPNL reduction of $3.3 \mathrm{~dB}$. The predicted noise reduction benefits from the ACTE flaps and MLG porous fairings are well within the range of values obtained during flight test of the same technologies. The shielding effect of the porous knee fairings helped reduce noise across the frequency range studied, with the largest effect observed in the mid-to-high frequency ranges. The tonal response of the various MLG cavities and subcomponents was also reduced through the use of the porous fairings.

\section{Acknowledgments}

This work was supported by the Flight Demonstrations and Capabilities (FDC) project under the Integrated Aviation Systems Program (IASP) of the NASA Aeronautics Research Mission Directorate. We would like to express our sincere appreciation to personnel at the NASA Armstrong Flight Research Center for their assistance with 
development of the full-scale G-III aircraft geometry, in particular Daniel Nolan and Michael Yandell of Jacobs Engineering for meticulously creating a high-definition CAD model for the main landing gear from the laser scan files. The authors also gratefully acknowledge the invaluable contribution of Scott Brynildsen of Craig Technologies, for providing geometry modifications and CAD support. All simulations were performed on the Pleiades supercomputer at the NASA Advanced Supercomputing (NAS) facility at Ames Research Center. The logistical support provided by NAS staff is greatly appreciated.

\section{References}

[1] M. Adib, F. Catalano and et al., "Novel Aircraft-Noise Technology Review and Medium- and Long-Term Noise Reduction Goals," International Civil Aviation Organization, vol. Doc 10017, 2014.

[2] W. Dobrzynski, "Almost 40 Years of Airframe Nosie Research: What Did We Achieve," J. Aircraft, vol. 47, no. 2, pp. 353367, March-April 2010.

[3] J. Appelbaum, B. Duda, E. Fares and M. R. Khorrami, "Airframe Noise Simulations of a Full-Scale Aircraft," paper to be presented at the AIAA/CEAS Aeroacoustics Conference, Atlanta, GA, June 2018.

[4] M. R. Khorrami, E. Fares and D. Casalino, "Towards Full-Aircraft Airframe Noise Prediction: Lattice-Boltzmann Simulations," AIAA Paper 2014-2481, June 2014.

[5] M. R. Khorrami and E. Fares, "Simulation-Based Airframe Noise Prediction of a Full-Scale Aircraft," AIAA Paper 20162706, May-June 2016.

[6] E. Fares, B. Duda and M. R. Khorrami, "Airframe Noise Prediction of a Detailed Full Aircraft in Model and Full Scale Using a Lattice Boltzmann Approach," AIAA Paper 2016-2707, May-June 2016.

[7] E. Baumann and E. Waggoner, "Flight and Ground Operations in Support of Airframe Noise Reduction Test," paper to be presented at the AIAA/CEAS Aeroacoustics Conference, Atlanta, GA, June 2018.

[8] M. R. Khorrami, D. P. Lockard, W. M. J. Humphreys and P. A. Ravetta, "Flight-Test Evaluation of Airframe Noise Mitigation Technologies," paper to be presented at the AIAA/CEAS Aeroacoustics Conference, Atlanta, GA, June 2018.

[9] S. Kota, P. Flick and F. Collier, "Flight Testing of the FlexFoil ${ }^{\mathrm{TM}}$ Adaptive Compliant Trailing Edge," AIAA Paper 20160036, January 2016.

[10] E. J. Miller, J. Cruz, S. F. Lung, S. Kota, G. Ervin and K. J. Lu, "Evaluation of the Hinge Moment and Normal Force Aerodynamic Loads from a Seamless Adaptive Compliant Trailing Edge in Flight," AIAA Paper 2016-0038, January 2016.

[11] C. Y. Herrera, N. D. Spivey and S. F. Lung, "Aeroelastic Response of the Adaptive Compliant Trailing Edge Transition Section," AIAA Paper 2016-0467, January 2016.

[12] M. R. Khorrami, W. M. Humphreys, Jr. and D. P. Lockard, "An Assessment of Flap and Main Landing Gear Noise Abatement Concepts," AIAA Paper 2015-2987, June 2015.

[13] H. Chen, "Volumetric Formulation of the Lattice-Boltzmann Method for Fluid Dynamics: Basic Concept," Physical Review $E$, vol. 58, no. 3, pp. 3955-3963, 1998.

[14] H. Chen, C. Texeira and K. Molvig, "Realization of Fluid Boundary Conditions via Discrete Boltzmann Dynamics," Int. Journal of Modern Physics C, pp. 1281-1292, 1998.

[15] H. Chen, S. Kandasamy, S. . Orszag, R. Shock, S. Succi and V. Yakhot, "Extended Boltzmann Kinetic Equation for Turbulent Flows," Science, vol. 301, pp. 633-636, 2003.

[16] H. Chen and G. Doolen, "Lattice Boltzmann Method for Fluid Flows," Annual Review of Fluid Mechanics, vol. 30, pp. 329364, 1998

[17] E. Fares and S. Noelting, "Unsteady Flow Simulations of a High-Lift Configuration Using Lattice-Boltzmann Approach," AIAA Paper 2011-869, 2011.

[18] D. Casalino, S. Noelting, E. Fares, T. Van de Ven, F. Perot and G. Bres, "Towards Numerical Aircraft Noise Certification: Analysis of a Full-Scale Landing Gear in Fly-Over Configuration," AIAA Paper 2012-2235, June 2012.

[19] D. Casalino, A. F. P. Ribeiro, E. Fares, S. Noelting, A. Mann, F. Perot, Y. Li, P. T. Lew, C. Sun, P. Gopalakrishnan, R. Zhang, H. Chen and K. Habibi, "Towards Lattice-Boltzmann Prediction of Turbofan Engine Noise," AIAA Paper 20143101, June 2014.

[20] H. Chen, S. Chen and W. H. Matthaeus, "Recovery of the Navier-Stokes equations using a lattice gas Boltzmann method," Physical Review A, no. 45, pp. R5339-T5342, 1992.

[21] Y. H. Qian, D. D'Humieres and P. Lallemand, "Lattice BGK Models for Navier-Stokes Equation," Europhysics Letters, vol. 17, pp. 479-484, 1992.

[22] V. Yakhot and S. Orszag, "Renormalization Group Analysis of Turbulence," Journal of Scientific Computing, vol. 1, no. 2, pp. 3-51, 1986. 
[23] E. Fares, "Unsteady Flow Simulation of the Ahmed Reference Body using a Lattice Boltzmann Approach," Journal of Computers and Fluids, no. 35, pp. 940-950, 2006.

[24] J. E. Hawkings and D. L. Ffowcs Williams, "Sound Generation by Turbulence and Surfaces in Arbitrary Motion," Philosophical Transactions of the Royal Society of London. Series A, Mathematical and Physical Sciences, vol. 264, no. 1151, pp. 321-342, 1969.

[25] G. Succi and F. Farassat, "The Prediction of Helicopter Discrete Frequency Noise," Vertica, vol. 7, no. 4, pp. $309-320$, 1983.

[26] A. Najafi-Yazdi, G. Bres and L. Mongeau, "An Acoustic Analogy Formulation for Moving Sources in Uniformly Moving Media," Proceedings of The Royal Society of London A, vol. 467, no. 2125, pp. 144-165, 2011.

[27] ICAO, "Environmental Protection, Annex 16 to the Convention on International Civil Aviation," vol. 1, no. Aircraft Noise, Appendix 2.

[28] J. W. Paulson, "Wind-Tunnel Investigation of a Fowler Flap and Spoiler for an Advanced General Aviation Wing," NAS A TN D-8236, 1976.

[29] W. C. Sperry, "Aircraft Noise Evaluation," FAA-NO-68-34, September 1968. 